\title{
The Organization of Extrinsic Neurons and Their Implications in the Functional Roles of the Mushroom Bodies in Drosophila melanogaster M eigen Kei Ito ${ }^{1,6,7}$ Kazumi Suzuki, ${ }^{2,3}$ Patricia Estes, ${ }^{4}$ Mani Ramasw ami, ${ }^{4,5}$ Daisuke Yamamoto, ${ }^{1,2}$ and Nicholas J. Strausfeld ${ }^{5}$ \\ ${ }^{1}$ Yamamoto Behavior Genes Project ERATO (Exploratory Research for Advanced Technology) Japan Science and Technology Corporation (JST) \\ Mitsubishi Kasei Institute of Life Sciences \\ 194 Machida-shi, Tokyo, Japan \\ ${ }^{2}$ Mitsubishi Kasei Institute of Life Sciences \\ 194 Machida-shi, Tokyo, Japan \\ ${ }^{3}$ Tokyo University of Agriculture and Technology \\ 183 Fuchu-shi, Tokyo, Japan \\ ${ }^{4}$ Department of Molecular and Cellular Biology \\ University of Arizona \\ Tucson, Arizona 85721 USA \\ ${ }^{5}$ Arizona Research Laboratories Division of Neurobiology \\ University of Arizona \\ Tucson, Arizona 85721 USA
}

\section{Abstract}

Although the importance of the Drosophila mushroom body in olfactory learning and memory has been stressed, virtually nothing is known about the brain regions to which it is connected. Using Golgi and GAL4-UAS techniques, we performed the first systematic attempt to reveal the anatomy of its extrinsic neurons. A novel presynaptic reporter construct, UAS-neuronal synaptobrevin-green fluorescent protein (n-syb-GFP), was used to reveal the direction of information in the GAL4-labeled neurons. Our results showed that the main target of the output neurons from the mushroom body lobes is the anterior part of the inferior medial, superior medial, and superior lateral protocerebrum.

${ }^{6}$ Present address: National Institute for Basic Biology, 444-8585 Okazaki-shi, Myodaiji-cho, Japan.

${ }^{7}$ Corresponding author.
The lobes also receive afferents from these neuropils. The lack of major output projections directly to the deutocerebrum's premotor pathways discourages the view that the role of the mushroom body may be that of an immediate modifier of behavior. Our data, as well as a critical evaluation of the literature, suggest that the mushroom body may not by itself be a "center" for learning and memory, but that it can equally be considered as a preprocessor of olfactory signals en route to "higher" protocerebral regions.

\section{Introduction}

Of the many functional roles ascribed to the insect mushroom body, two dominate the literature. One is the mushroom body's role in olfactory processing, suggested in many species by its relationship with antennal lobe olfactory neuropils. The other is the mushroom body's supposedly crucial role in learning and memory. This is suggested by a number of studies that have either ascribed

LEARN IN G \& M EM ORY 5:52-77 @ 1998 by Cold Spring Harbor Laboratory Press ISSN 1072-0502/98 \$5.00

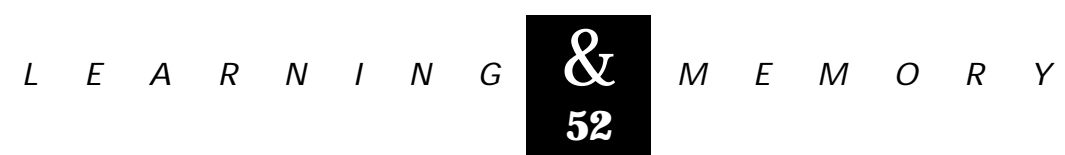


biochemical defects in olfactory learning and memory to the mushroom bodies (for review, see Davis 1993), or which have show $n$ that anatomical defects in the mushroom bodies (Heisenberg 1980; Heisenberg et al. 1985), even to the extent of their complete absence (de Belle and Heisenberg 1994), can impair or abolish olfactory learning and memory. In addition to these roles, there have been reports that mushroom bodies are involved in courtship behavior (Hall 1979; Ferveur et al. 1995; O'Dell 1995) and in place memory (Mizunami et al. 1993). Other studies have demonstrated that changes in mushroom body volume and/or fiber numbers are associated with specific behavioral tasks, especially in species that have multiethism (Balling et al. 1987; Withers et al. 1993, 1995; Durst et al. 1994; Heisenberg et al. 1995; Gronenberg et al. 1996; Barth and Heisenberg 1997). Elevated levels of expression in mushroom body cells have been observed for various genes associated with learning-related biochemical cascades (Nighorn et al. 1991; P.L. Han et al. 1992; Skoulakis et al. 1993; K.-A. Han et al. 1996; Skoulakis and Davis 1996; Muller 1997; Grotewiel et al. 1998). Some recent publications thus embrace the notion that the mushroom bodies are the center of learning and memory (P.L. Han et al. 1992; Tettamanchi et al. 1997).

How ever, there has been no direct demonstration, to date, that mushroom bodies are the site at which associative memory is formed, and where long-term changes in connectivity are established as a result of these associations. If the mushroom bodies are immediate behavioral modifiers that conditionally gate systems of descending neurons and motor neurons (Han et al. 1996), direct connection of the mushroom body output neurons to descending pathways might well be observed. Studies on large Brachyceran flies, such as Musca domestica, Calliphora erythroœphala, and Sarcophaga carnaria (Milde and Strausfeld 1990; Strausfeld and Gronenberg 1990), demonstrate that most of the descending neurons have their dendrites restricted to the ventrolateral and dorsolateral deutocerebrum, the latter also known as the posterior slope. The only exceptions are two pairs of small descending neurons that have dendrites within the superior medial protocerebrum (Gronenberg et al. 1995). There is a high degree of homology between descending neurons among Diptera (King and Valentino 1983), and descriptions of descending neurons in Drosophila (Strausfeld and Bassemir 1983; Phelan and Bacon 1997) also demonstrate that their dendrites are constrained to the lateral and dorsolateral deutocerebrum. If mushroom bodies modify behavior by modulating directly the activity of descending neurons, their outputs should be expected to project to these areas. Indeed, two such neurons were reported in the brain of the blow fly Calliphora erythrocephala (Strausfeld et al. 1984), and circuit models employing such direct connection have been proposed (Mizunami et al. 1993; Yang et al. 1995).

It is, however, not proven whether these two neurons represent the major pathw ay of the mushroom body output. In honeybees, extrinsic neurons are found to project from the mushroom body lobes to a variety of brain areas, including the mushroom body's ow n calyces (Gronenberg 1987; Rybak and Menzel 1993). The little that has been published about descending neurons does not suggest that their dendrites are visited by mushroom body efferents. Likewise, output neurons of the mushroom bodies of the cockroach Periplaneta americana terminate mainly in regions of the lateral and frontal protocerebrum with few, if any, direct associations with descending pathways ( $\mathrm{Li}$ and Strausfeld 1997a). In blow flies, as well as in Drosophila, two clusters of dopamine- and tyrosine hydroxylase-immunoreactive neurons are known to connect the mushroom body lobes to the protocerebrum (Nässel and Elekes 1992). If such connections to the protocerebrum comprise the major output pathway, the role of the mushroom bodies as the center for association would remain rather ambiguous. Considering the elaborate neural netw ork existing in these protocerebral regions, it is not clear whether learning and memory are the property of the mushroom bodies themselves or that of yet higher protocerebral "centers" to which the mushroom bodies transmit information.

For the Drosophila nervous system, the projection patterns from the sensory organs to the primary sensory neuropils, and from there to the mushroom bodies, have been documented in great detail (summarized in Fig. 1, below; see also Stocker et al. 1990; Stocker 1994). Compared to this, our know ledge is very limited about the exact area of neuropil to which the mushroom bodies convey information. To address this question, we performed the first systematic attempt to reveal the morphologies and projections of extrinsic neurons of the Drosophila mushroom bodies. Using Golgi and GAL4-UAS techniques, we found that

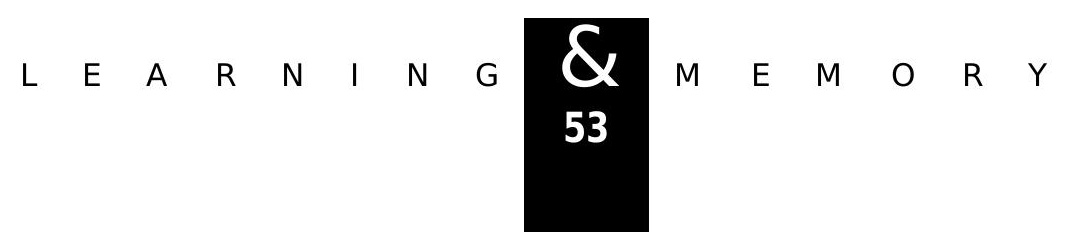


Ito et al.

the majority of these neurons invade the anterior portion of the inferior medial, superior medial, and superior lateral protocerebrum, which are still far removed from the descending pathways. This suggests that direct connections to descending neurons are not the major pathways of the mushroom body outputs. The implications of this organization for studies on Drosophila learning and memory are discussed.

\section{Materials and Methods}

\section{FLY STOCKS AND DNA CONSTRUCTS}

The Canton special (CS) strain was used to represent the wild type. The GAL4 enhancer-trap strains were obtained from a previous screening by G.M. Technau's group at University of Mainz (Ito et al. 1995) and by K. Suzuki at the Mitsubishi Kasei Institute of Life Sciences (Ito et al. 1997a). Both series were made by crossing a pGaw $B$ strain (Brand and Perrimon 1993) with the $\mathrm{P}\left[\mathrm{ry}^{+} ; \Delta 2-3\right]$ (99B) strain (Robertson et al. 1988). To reveal the GAL4 expression pattern, male GAL4 flies were crossed with female flies carrying the microtubuledirected UAS-tau (Ito et al. 1997b), cytoplasmic UAS-GFP S65T (a variant of green fluorescent protein: see Ito et al. 1997a; B. Dickson, unpubl.), nuclear-targeted UAS-NLS-lacZ (nuclear localization signal-lacZ fusion; Y. Hiromi, unpubl.), or presynaptic UAS-n-syb-GFP (neuronal-synaptobrevin-GFP S65T fusion) constructs.

For generating flippase-mediated clones, female flies homozygous for hsp70-flp (Golic and Lindquist 1989) were crossed to males homozygous for both AyGAL4 and UAS-tau (Ito et al. 1997a). Clones were generated by giving mild heat shock at the second larval instar, when most of the postembryonic neuroblasts in the central brain have become active mitotically (Ito et al. 1992, 1997a). Mature female flies $>5$ days after eclosion were used for histology, except for the flippase clones, in which case female flies just after eclosion were used.

The GAL4-responsive n-syb-GFP transgene was generated by Pelement-mediated transformation with a construct in which the GFP S65T was fused in-frame to the lumenal carboxyl terminus of a Drosophila synaptic-vesicle membrane-protein neuronal synaptobrevin (DiAntonio et al. 1993), which is the homolog of vertebrate vamp (Trimble et al. 1988). This fusion protein, when expressed in identified Drosophila motor neurons, has been show $\mathrm{n}$ to be transported to nerve terminals where it is restricted to presynaptic varicosities, in a pattern indistinguishable from that of synaptic vesicles. Furthermore, when examined in the background of Kinesin heavy chain (Khc), or shibire ${ }^{\text {ts }}$ (shits) mutants (Estes et al. 1996; Hurd and Saxton 1996), in which synaptic-vesicle membrane proteins are redistributed, an identical redistribution of n-syb-GFP is observed (P.E. Estes, G. Ho, D. Hurd, W. Saxton, and M. Ramaswami, in prep.). Detailed description of these data, demonstrating that n-syb-GFP is a bona fide marker for synaptic vesicles at nerve terminals, are described elsewhere (P.E. Estes, G. Ho, D. Hurd, W. Saxton, and M. Ramaswami, in prep.). Without GAL4 driver, the UAS-n-syb-GFP strain used in this study shows no labeling in the brain neuropil (Fig. 5K, below). In the cortex, occasional dots are observed. These random dots appear also in the presence of GAL4, but their pattern is not symmetrical and does not correspond to the positions of the GAL4-expressing cell bodies (e.g., cf. Fig. 5, A and B, C and D, and $E$ and $F$, below). Thus, these cortical dots can be disregarded as noise when observing the staining pattern in the neuropil.

\section{STAINING}

Bodian (Fig. 2A-C) and Golgi impregnations (Figs. 2D-G and 3A-H, below) were made on cold anesthetized Drosophila, threaded betw een adjustable gelatin sheets that substitute for the metal blades of disposable Teflon staining collars originally described by Jäger and Fischbach (1987). For Golgi impregnations (I. Vilinsky and N.J. Strausfeld, in prep.), collar and flies were submerged in five parts $2.5 \%$ potassium dichromate and one part $25 \%$ EM-grade glutaraldehyde (Electron Microscopy Sciences, Ft. Washington, PA). The cuticle between the eyes and mouth parts was removed. Addition of 2 grams chloral hydrate $/ 100 \mathrm{ml}$ solution favored impregnation of glial cells. After 5 days, tissue was washed in $2.5 \%$ potassium dichromate and immersed for 4 days in 99:1 parts of $2.5 \%$ potassium dichromate and $1 \%$ osmium tetroxide. The staining collar with its attached flies was then dipped in distilled water and placed in $0.75 \%$ silver nitrate for 3 days. The entire procedure ran at $4^{\circ} \mathrm{C}$. After dehydration through propylene oxide, collars were inverted over a BEEM capsule filled with Durcupan (Fluka, Heidelberg, Germany). After plastic infiltration and polymerization, the collar was snapped off from the block, leaving a row of em-

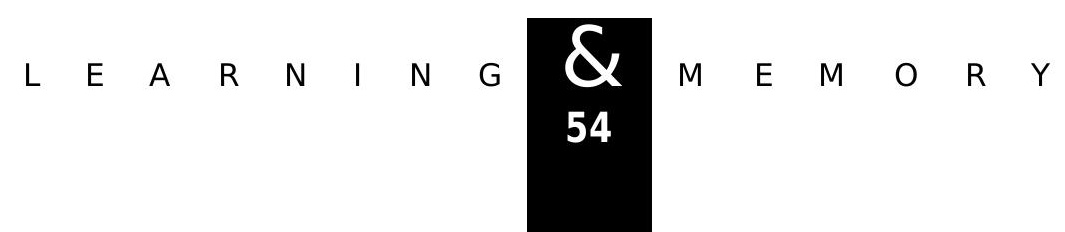


bedded heads for serial sectioning at 12-15 $\mu \mathrm{m}$. For Bodian silver staining, heads were fixed in AAF ( $80 \mathrm{ml}$ 98\% ethanol, $5 \mathrm{ml}$ glacial acetic acid, $15 \mathrm{ml}$ $37 \%$ formalin). Serial $10-\mu m$ Paraplast sections were stained according to Bodian's (1936) original method.

For whole-mount antibody staining of enhancer-trap strains (Figs. 4 and 6, below), brains were dissected in PBS and fixed with 4\% EM-grade formaldehyde in PEM buffer for 50 min (Ito et al. 1995). The mouse monoclonal anti-Tau primary antibody (Sigma, diluted at 1:500), biotin-conjugated secondary antibody (Vectastain, 1:500), and Vectastain Elite $A B C$ kit for $D A B$ staining were used to reveal cells labeled with UAS- tau (Ito et al. 1997b). Preparations were embedded in Araldite and kept in 0.3- to $0.35-\mathrm{mm}$ capillaries, which can be rotated under microscope objectives (Prokop and Technau 1993).

For confocal microscopy (Figs. 5, 7, and 9, below), the GFP-labeled brains were dissected in PBS and fixed with $4 \%$ formaldehyde/PEM for $50 \mathrm{~min}$, washed with PBS, and mounted in $50 \%$ glycerol/ PBS containing $0.25 \% \mathrm{n}$-propyl gallate as an antifade. To reveal cells labeled with UAS-NLS-lacZ, the mouse monoclonal anti- $\beta$ galactosidase primary antibody (Promega, diluted at 1:500) and FITC-conjugated secondary antibody (Amersham, 1:50) were used.

\section{IMAGING}

Camera lucida drawings of Golgi-impregnated neurons and glia (Fig. 3, below) were made at a final magnification of $2000 x$, scaled, and superimposed on perimeters of Bodian-stained images, using Smartsketch (Futurew are, San Diego, CA). Images for Figure 2 were captured using a Sony DC 5000 digital camera, merging up to six successive optical sections using Adobe Photoshop 4.0 softw are (Adobe Systems, San Jose, CA). Digital camera lucida images of Golgi-impregnated Kenyon cells (Fig. 2A, below) were scaled and superimposed on Bodian-stained sections at the relevant level.

Digital camera lucida images of whole-mount brains (Figs. 4 and 6, below) were composed by using Adobe Photoshop software and a Macintosh computer to montage 20-70 photographs captured at slightly different focal planes with a Kontron Progress 3012 digital scanning camera mounted on a Zeiss Axiophot microscope equipped with $40 \times$ and $63 \times$ Nomarski optics. Unlike ordinary montages (e.g., Fig. 2D-G, below), only the cells of interest were traced, others being obscured during the montage process [e.g., cf. Fig. $6, E$ and $G$ (below), which are composed from the same preparation]. Despite their photographic appearance, these pictures should therefore be regarded as "camera lucida images" rather than photographs.

Throughout this paper, horizontal views are show $\mathrm{n}$ with anterior to the bottom. Though unconventional, this orientation provides better comparison of the frontal, oblique, and horizontal views, and is traditionally used for drafting first- and thirdangle projections. This also allows us to show the mushroom body in its natural orientation.

For confocal microscopy, 120-140 serial optical sections at 1- to $1.3-\mu \mathrm{m}$ intervals were taken with 40x objective and reconstructed using a Sarastro $2000 \mathrm{Z}$ laser confocal microscope and ImageSpace software (Molecular Dynamics, Sunnyvale, CA).

The original images reported in this paper have been submitted to the FLYBRAIN image database (http: // www.flybrain.org) under the accession number AD01010.

\section{TERMINOLOGY}

The terms $\alpha$ and $\beta$ lobes (Vowles 1955) refer to the vertical and medial branches of the mushroom bodies, arising from the pedunculus. They are observed in all insect species described so far (for review, see Strausfeld 1998). In Lepidoptera and Diptera, which includes Drosophila, the medial branch comprises two divisions, the $\beta$ lobe and a second larger component called $\gamma$ (Pearson 1971). The $\beta$ lobe lies immediately behind $\gamma$. The vertical lobe in Drosophila also comprises two components called $\alpha$ and $\alpha^{\prime}$ (Ito et al. 1997a). The $\alpha$ lobe is taller, and its head is inclined slightly medially (Figs. 1 and 2, A and B, below). The $\alpha$ and $\beta$ lobes share axon collaterals from one subclass of Kenyon cells, which are the intrinsic neurons of the mushroom bodies (Fig. 3B, right, below). The $\alpha$ ' lobe, originally described as "wedge-shaped thickening along the $\alpha$ lobe" (see plate I in Heisenberg 1980), is shorter, and its head bends slightly laterally (Figs. 1 and 2, A and B). The $\alpha^{\prime}$ and $\gamma$ lobes share axon collaterals from another class of Kenyon cells (Fig. 3A, left, below; see Fig. 7 in Ito et al. 1997a). For convenience, the generic term "vertical lobes" will be used when describing the ensembles of $\alpha$ and $\alpha$, and "medial lobes" for $\beta$ and $\gamma$ combined.

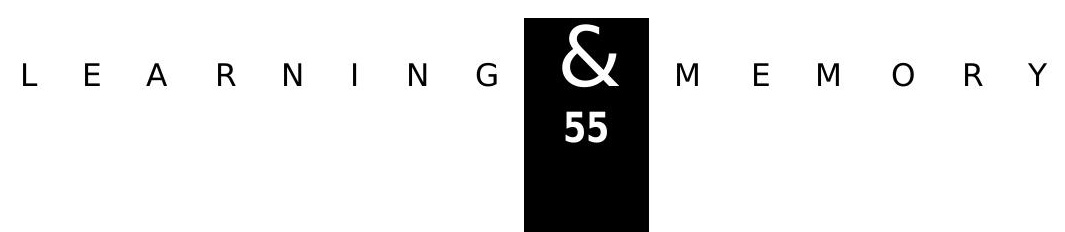


Ito et al.

Figure 1: Chemosensory-related neuropils in the brain (anterior oblique frontal view). Approximately 1200 antennal olfactory neurons (Stocker et al. 1990) project via the antennal nerve (ant $n$ ) to the antennal lobe (ant lob) on each side of the esophageal foramen (oes). Approximately 125 olfactory neurons from the maxillary palps and 195 gustatory neurons from the labial palps enter the gustatory center of the subesophageal ganglion via the labial nerve $(\mathrm{lb} n)$, and project to the antennal lobe via the antenno-subesophageal tract (AST; N ayak and Singh 1983; Singh and Nayak 1985). Three gustatory axons from the dorsal cibarial sense organ project to the gustatory center via the pharyngeal nerve (phy n). Two to four axons from the ventral cibarial sense organ and 10 axons from the labral sense organ project via the

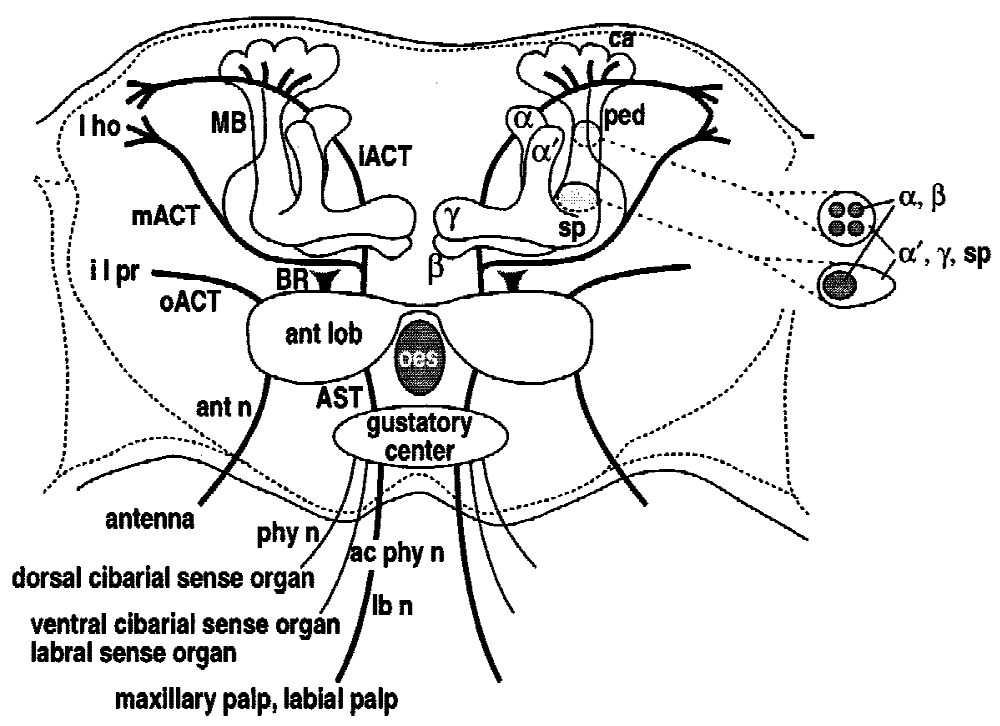
accessory pharyngeal nerve (ac phy $\mathrm{n}$ ). The ventral nerve cord (not shown) receives projections from 150-200 gustatory neurons in each foreleg, 120 in the middle and hind legs, 160 from each wing, and female genitalia (Stocker 1994). Terminals from the antennae and maxillary and labial palps form 43 antennal lobe glomeruli (R. Stocker, pers. comm.). About 200 output fibers from the antennal lobe project through the inner and middle antennocerebral tract (iACT, mACT; Stocker et al. 1990). The iACT projects to the lateral horn (I ho), sending collaterals to the mushroom body calyx (ca). The mACT projects directly to the lateral horn with a small subset of fibers entering the pedunculus and terminating in the calyx. The outer antennocerebral tract (OACT) contains a small number of fibers connecting the ant lob with the inferior lateral protocerebrum (i I pr). The ill-defined tract of Power's (1946) broad root (BR) contains, at most, a few fibers projecting posteriorly from the ant lob. 2500-3000 Kenyon cells (Hinke 1961; Balling et al. 1987) form a quadruple structure of clonally related units (Ito et al. 1997a) providing dendrites to the calyces and sending axons into the pedunculus (ped). The core of the ped contains four bundles of axons, which anteriorly coalesce and bifurcate into the $\alpha$ and $\beta$ lobes. Surrounding this core are axons that contribute to the spur (sp) and $\alpha^{\prime}$ and $\gamma$ lobes.

Figure 2: Bodian and Golgi stained mushroom bodies. $(A-C)$ Bodian-stained frontal sections showing one mushroom body's medial and vertical lobes $(A, B)$, and pedunculus cross section $(C)$. (s $\mathrm{m} \mathrm{pr}$ ) Superior medial protocerebrum, ( $\mathrm{s}$ pr) superior lateral protocerebrum, ( $\mathrm{m} \mathrm{pr}$ ) inferior medial protocerebrum, ( $\mathrm{I}$ pr) inferior lateral protocerebrum. ( $\mathrm{m}$ bdl) M edian bundle, (ant lob) antennal lobe. (A) O utline (red) indicates limits of mushroom body neuropil. The head of the $\gamma$ lobe has three swellings ( $\gamma 1-\gamma 3$ from dorsal to ventral; see also $D)$. The middle swelling $(\gamma 2)$ lies more posterior to $\gamma 1$ and $\gamma 3$. The keel $(\mathrm{ke})$ is a local swelling within the $\gamma$ lobe, and the spur (sp) is the root swelling of $\gamma$. The $\beta$ lobe lies ventroposterior to $\gamma$. Its terminal sw elling bends downwards and situates behind $\gamma 3$ and beneath $\gamma 2$ (see also Fig. 4E). Note the taller, medially inclined $\alpha$ lobe and the lower, laterally bent $\alpha^{\prime}$ lobe. Golgi-impregnated Kenyon cell axons are superimposed against the Bodian stained lobes. Axons extending through $\gamma$ have short branches to the spur (sp) and long collaterals to $\alpha^{\prime}$. Thinner, straighter axons in $\beta$ have collaterals into $\alpha$. (B) Profiles of extrinsic neurons within the root of the vertical lobe (left arrow ex) and the swellings of the $\gamma$ lobe (right arrow ex). Argyrophilic extrinsic neuron profiles in the head of $\gamma$ match profiles of Golgi-impregnated extrinsic neurons in Fig 3D, right. Glial cell bodies (gl) reside at the edges and within the mushroom body neuropil (montage of three optical sections). (C) Pedunculus cross section near its anterior end (ped) contains few argyrophilic extrinsic fibers (upper left, arrow ex). Extrinsic fibers (upper right, two arrows ex) connect the posteriormost $\gamma$ lobe swelling $(\gamma 2)$ and surrounding neuropil. Processes linking the left and right $\beta$ lobes (lower right, arrow bi ex) correspond to connections of bilateral extrinsic neurons in $F, G$, and Fig. 3G. (D) Three swellings of the $\gamma$ lobe head. In this Golgi preparation $\gamma 3$ receives a subset of Kenyon cell axons alone. The more dorsal $\gamma 1$ and $\gamma 2$ swellings show convergence of Kenyon cell axons and an afferent terminal. $(E)$ Bilateral terminal of an afferent supplying $\gamma 2$ (complete reconstruction; Fig. 3F). (F) H eterolateral extrinsic neuron dendrites connect terminal sw ellings of the $\beta$ lobes (see Fig. 3G). A whorl of Kenyon cell axons is labeled in $\gamma 2$. (G) Another preparation reveals the same type of extrinsic neuron as in $F$, suggesting morphological consistency in different individuals. Scale bar, $25 \mu \mathrm{m}$.

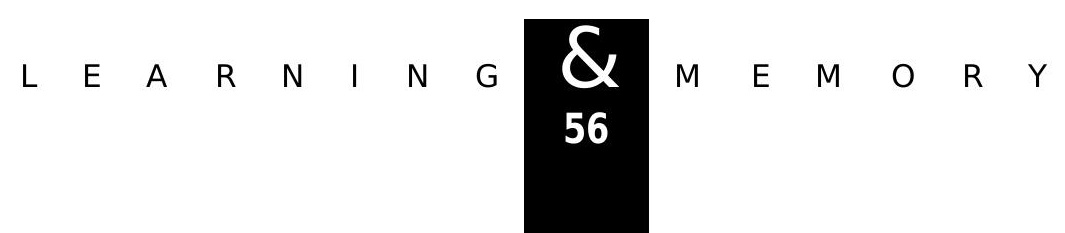



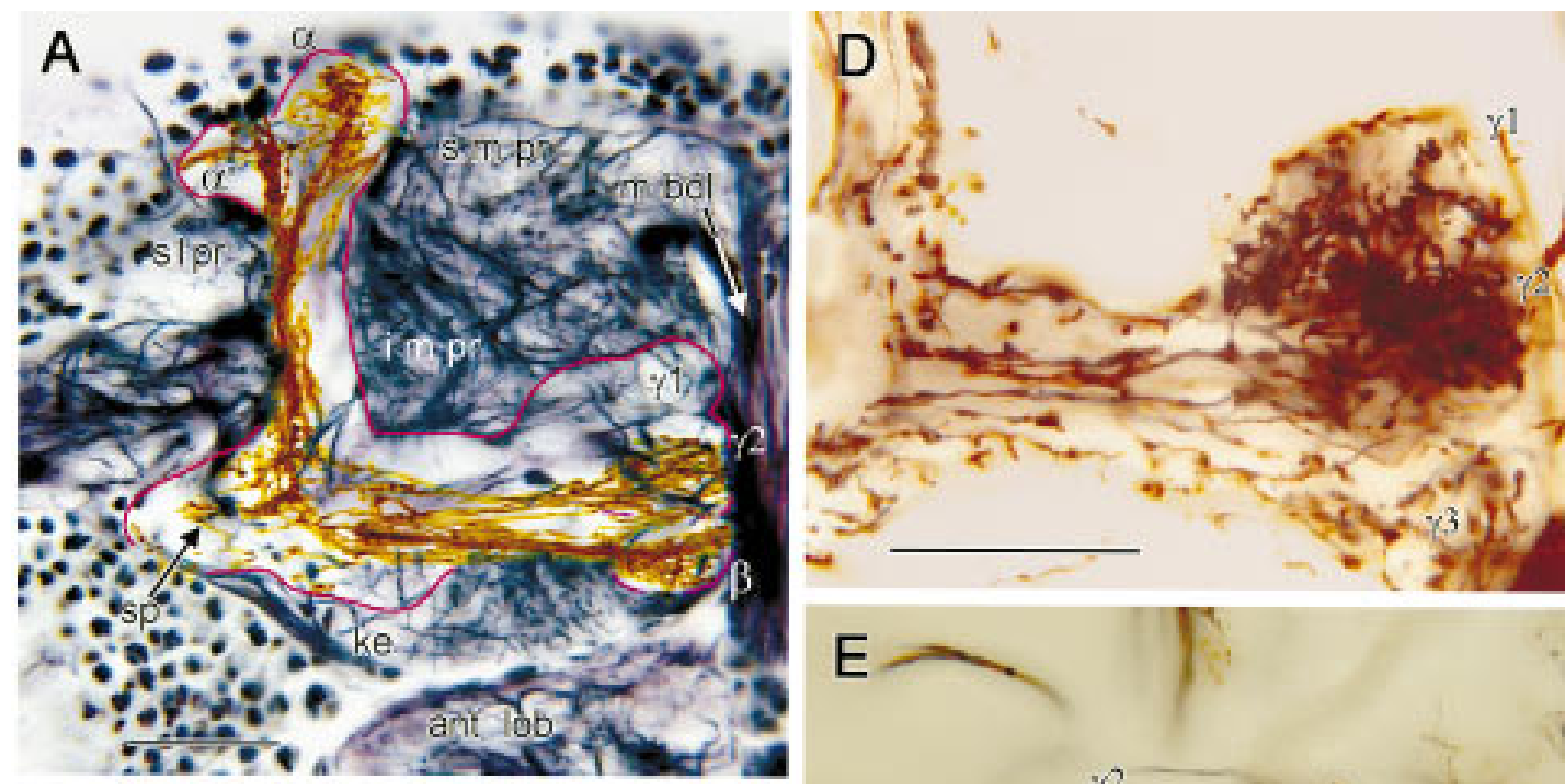

\section{B}

B
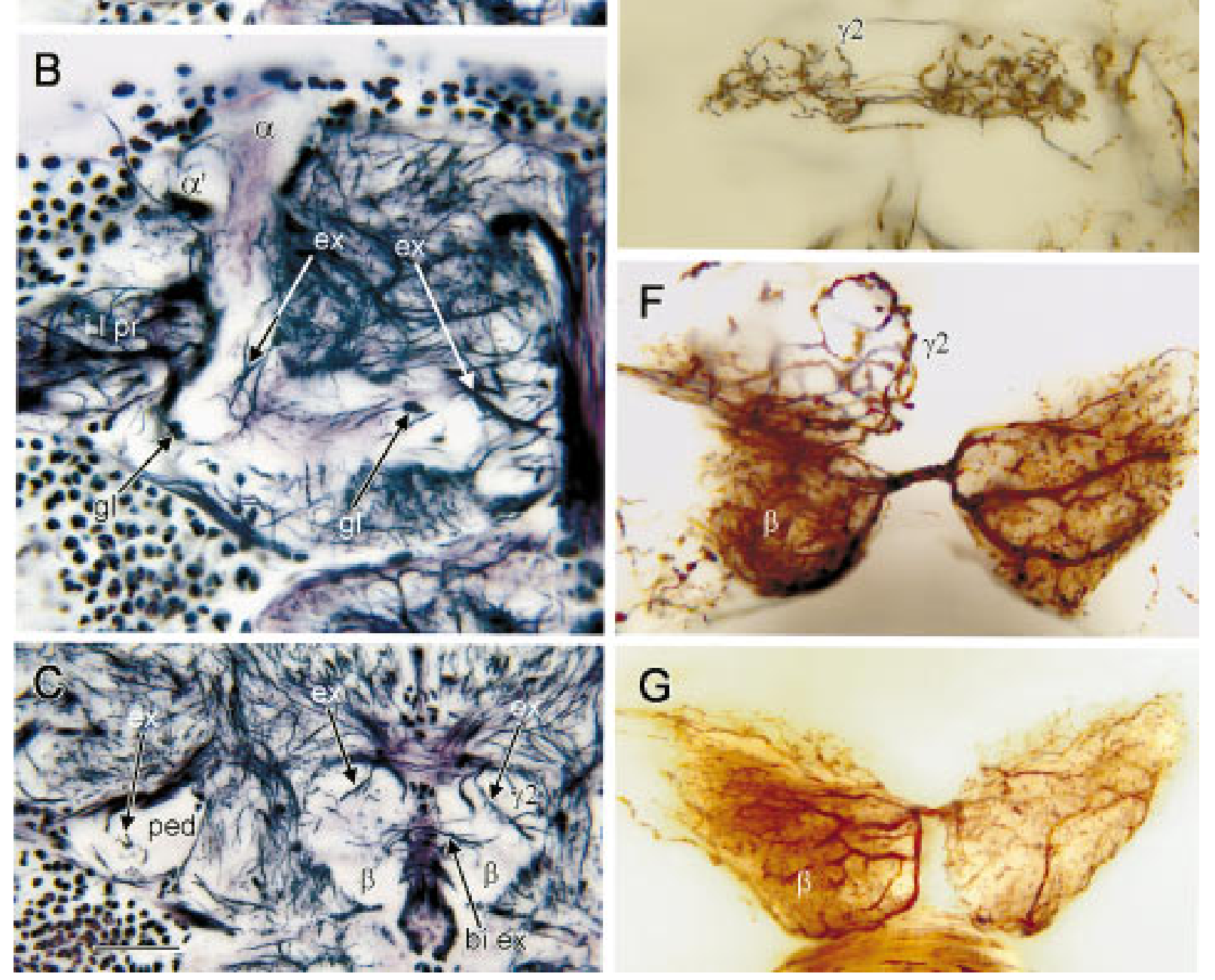

Figure 2: (See facing page for legend.)

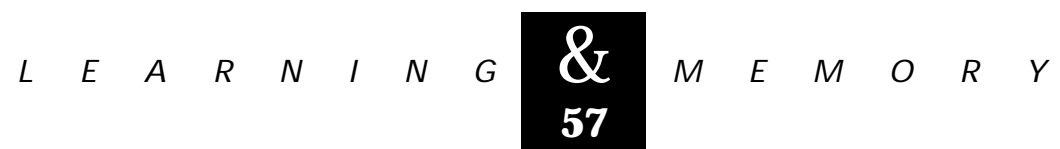


Ito et al.

The term "extrinsic neuron" (Mobbs 1982) is conferred on neurons (other than afferents to the calyces) that have processes in the mushroom bodies and projections extending to (efferents) or from (afferents) other brain regions. "Output neurons" are here referred to as mushroom body efferent neurons.

The term cortex, synonymous with the term rind, refers to the layer of cell bodies that covers the neuropil. The thickness may vary from as thin as a glial sheath with sparse neuronal cell bodies to a thick aggregate of cell bodies such as the area surrounding the mushroom body calyces. In general, synapses are not observed in the insect cortex.

\section{Results}

\section{EXTRINSIC NEURONS REVEALED BY GOLGI AND BODIAN STAINING}

Bodian staining reveals profiles of large processes in the mushroom body as well as in the fibroarchitecture of the surrounding protocerebrum, in conjunction with the pale background staining of the mushroom body neuropil (Fig. 2AC). This is attained by the fact that the Kenyon cells are too small to be revealed individually by this technique, which stains neurotubular protein. Superimposition of Golgi-impregnated Kenyon cell axons onto Bodian-stained mushroom bodies (Fig. $2 \mathrm{~A})$ reveals the segregation of axons into the two components of the vertical lobe: $\alpha$ and $\alpha^{\prime}$. Cell bodies of glial cells are found lying adjacent to or within the mushroom body neuropils (Fig. 2B; see also Fig. 3C, right).

Moving from the front of the brain (Fig. 2A,B) some $25 \mu \mathrm{m}$ tow ards the back (Fig. $2 \mathrm{C}$ ), reveals the terminal swelling of the $\beta$ lobe, beneath the pos- teriormost (middle) swelling of $\gamma(\gamma 2)$. Most extrinsic processes can be seen in the sw ellings of the $\gamma$ lobe. Some profiles, such as that indicated by the right arrow (ex) in Figure 2B, match efferent neurons, such as illustrated in Figure $3 C$ (left). Profiles indicated by the left arrow (ex) in Figure 2B correspond to the trajectories of efferent neurons such as the one shown in Figure 3D (left). The bulbous tips of the $\beta$ lobes are linked heterolaterally by argyrophilic axons (bi ex in Fig. 2C). These correspond to heterolateral extrinsic neurons revealed by the Golgi method (Figs. 2, F and G, and $3 G)$. The vertical lobes ( $\alpha$ and $\alpha^{\prime}$ ) reveal sparse extrinsic processes, if any, as does the pedunculus (shown as cross section to the left in Fig. 2C).

Golgi impregnations demonstrate that extrinsic neuron dendrites occupy characteristic zones or segments within the lobes. They are located where the axons of Kenyon cells provide clusters of specializations (spines or varicosities) or where they form whorls, as in the terminal swellings of $\gamma$ and $\alpha^{\prime}$, or $\beta$ and $\alpha$. For example, the arborization of one extrinsic neuron, shown impregnated with axons of clawed Kenyon cells in Figure 2D, occupies the dorsal sw ellings of the bulbous head of the $\gamma$ lobe $(\gamma 1$ and $\gamma 2$ ). Another extrinsic neuron arbor, shown in Figure 2E (and in Fig. 3F), sends varicose processes only to the second sw elling of $\gamma$ $(\gamma 2)$ heterolaterally and a recurrent process into the keel of $\gamma$, and into the base of the vertical lobe (Fig. 3F). The extrinsic neuron show $n$ in Figure $3 \mathrm{C}$ (left) has processes in all three sw ellings of $\gamma$ and a recurrent branch that extends to a zone of branchlets and spines that arise from Kenyon cell axons located above $\gamma$ 's keel.

In Figure 3B (right), dendrites of the pinnate Kenyon cells are located $w$ ithin the core of the $\beta$ lobe. The neuron shown in Figure 3B (left) has

Figure 3: Camera lucida drawing of Golgi impregnated mushroom body neurons against neuropil outlines. ( $A$, left) Kenyon cells with clawed dendrites in the calyx (ca) send axons through the pedunculus (ped) to $\gamma$ and $\alpha^{\prime}$ lobes where they form whorls within terminal swellings. ( $A$, right) Antennal lobe (ant lob) projection neurons comprise the major supply to the calyx (ca) via the inner antennocerebral tract (i act), extending into the lateral horn (I ho). ( $B$, left) Efferent neuron from the $\beta$ lobe, with terminals in $\alpha$ and in surrounding s $\mathrm{m}$ pr. ( $B$, right) Kenyon cells with slender spined dendrites in the calyx supply the $\beta$ and $\alpha$ lobes. $(C$, left) An efferent neuron originating from terminal swellings of $\gamma$ sends recurrent branches back into $\gamma$ and an axon to the head of $\alpha^{\prime}$ and surrounding $\mathrm{s} \mathrm{m} \mathrm{pr.} \mathrm{(C,} \mathrm{right)} \mathrm{Glial} \mathrm{cells} \mathrm{in} \gamma$ swellings, in the ped- $\alpha^{\prime}$ junction near the spur (sp), and at the head of $\alpha^{\prime}$ occupy distinct domains corresponding to specific extrinsic neuron processes. $(D$, left) Efferent neuron arising from the pedunculus- $\alpha$ lobe junction, with terminals in the roof of the $s \mid \mathrm{pr}$ and part of the $\mathrm{s} \mathrm{m}$ pr. $(D$, right) Efferent neuron from terminal swellings of $\gamma$, with endings in the $\mathrm{s} \mathrm{m}$ pr and $\mathrm{i} \mathrm{m}$ pr. $(E)$ Protocerebral "interior" neuron supplying heterolateral arborizations to $\gamma$ and unilateral branches to the head of $\alpha$ '. (F) Afferent extrinsic neuron with dendrites (de) in the i $\mathrm{m} \mathrm{pr}$, providing heterolateral terminals to dorsal swellings of $\gamma$ (see Fig. 2E), and recurrent endings in the keel (ke) of $\gamma .(G)$ Heterolateral efferent neuron originating from the terminal sw elling of $\beta$ and providing varicose terminals in the i $\mathrm{m} \mathrm{pr}$, flanking the $\alpha$ lobe shaft. $(H)$ Heterolateral neuron linking the heads of $\alpha$, with arborizations in the $\mathrm{s} \mathrm{m}$ pr. Cell body location uncertain.

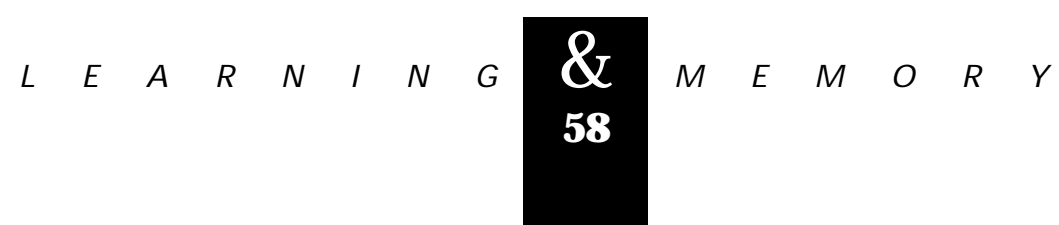




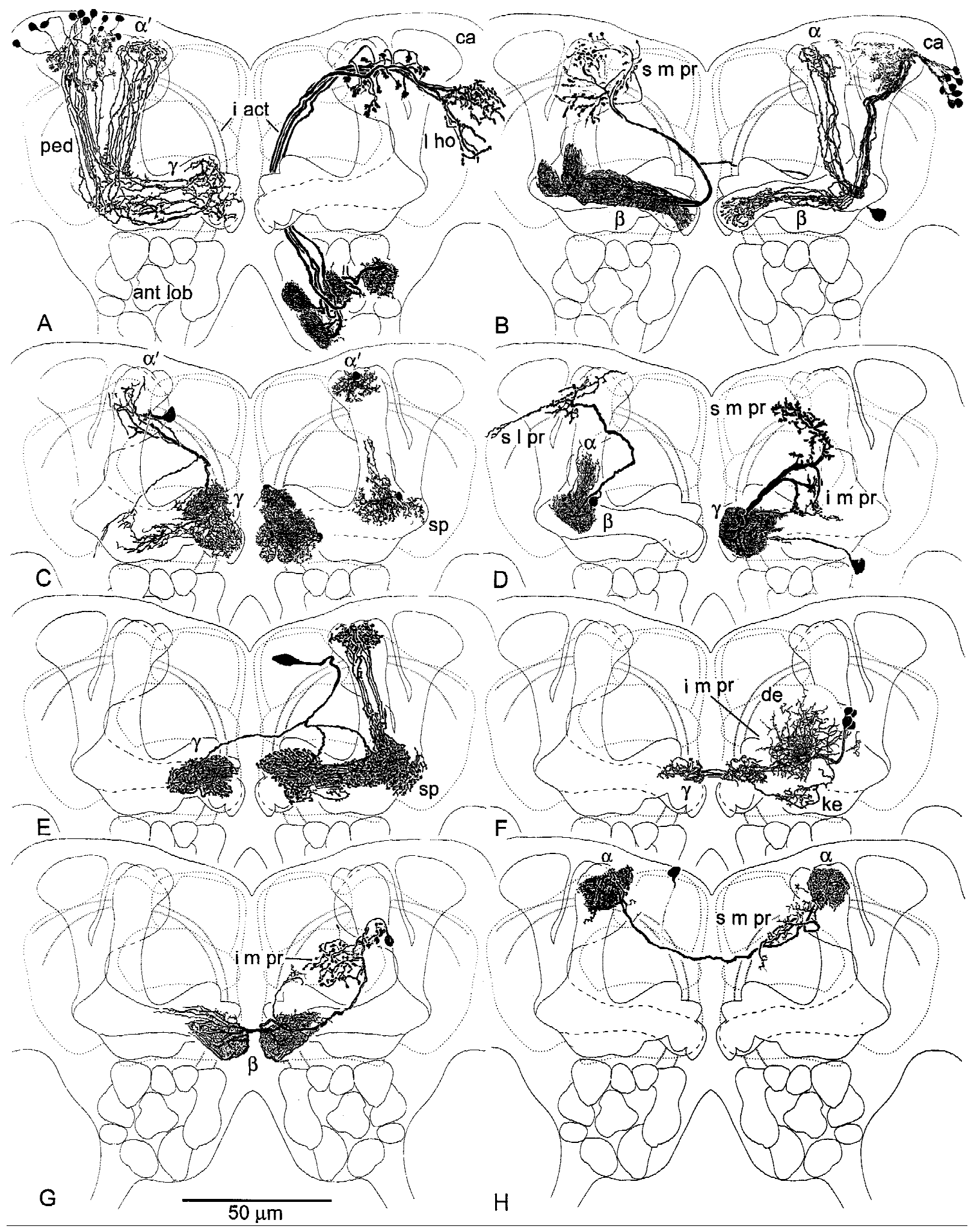

Figure 3: (See facing page for legend.)

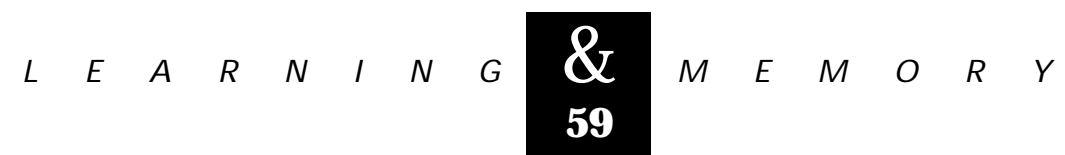


Ito et al.

dendrites that correspond to the length of the $\beta$ lobe, and two tufts which follow the axons of these pinnate Kenyon cells part of the way back into the base of the pedunculus, and part of the way up the shaft of $\alpha$. The cell body fiber of this neuron arises contralaterally. Heterolateral extrinsic neurons (Figs. 2, F and $G$, and $3, G$ and $H$ ) have dendrites constrained to the terminal swellings of their respective lobes.

Most of the extrinsic neurons described here are connected to regions of the superior medial protocerebrum (s $\mathrm{m} \mathrm{pr}$ ), superior lateral protocerebrum ( $s \mid \mathrm{pr}$ ), and inferior medial protocerebrum (i $\mathrm{m}$ pr). No neurons have been observed to project from the Drosophila mushroom bodies to the posterior slope in the deutocerebrum, which, by analogy with larger brachyceran Diptera, comprises regions that contain the dendrites of premotor descending neurons.

Examination of $\sim 500$ Golgi-impregnated brains revealed a total of 16 elements that could be identified as extrinsic cells. Of these, eight types were reliably distinguished (Fig. 3). The types of neurons invading the medial lobes (e.g., Fig. 3F,G) are observed more frequently than those invading the vertical lobes (e.g., Fig. 3H). Bodian staining also reveals more extrinsic fibers in the medial lobes than in the vertical lobes. Golgi preparations revealed no extrinsic neurons that invade the pedunculus, except near its anteriormost end. Bodian staining reveals few extrinsic fibers in the pedunculus cross section (Fig. 2C). Thus, although the Golgi method is stochastic, it is likely that there are more extrinsic neurons associated with the medial lobes than with the vertical lobes, and that the pedunculus contains few if any extrinsic neurons.

\section{EXTRINSIC NEURONS REVEALED BY GAL4-UAS SYSTEM}

Tw o methods featuring the GAL4-UAS system can label specific subsets of brain cells. The GAL4 enhancer-trap system (Brand and Perrimon 1993) labels cells that share common enhancer activity. The flippase (flp)-mediated FRT-GAL4 system (Ito et al. 1997a), on the other hand, labels cells that belong to the same lineage. We screened $\sim 1000$ preparations of postembryonic flippase clones and 500 GAL4 enhancer-trap strains. Compared to the number of clones and strains that labeled mushroom body intrinsic neurons (Kenyon cells), those that labeled extrinsic neurons were relatively scarce. We have so far identified only one flp clone and six enhancer-trap strains.

A major advantage of the GAL4-UAS system is that different aspects of labeled cells can be visualized by using appropriate reporter constructs. The microtubule-directed UAS-tau (Ito et al. 1997b) and cytoplasmic UAS-GFP S65T (green fluorescent protein: see also Ito et al. 1997a; B.

Figure 4: Extrinsic neurons connecting the mushroom body and medial protocerebrum. $A-H$ are digital camera-lucida montages (see Materials and Methods) of identical preparations viewed frontally (dorsal to the top) and horizontally (anterior to the bottom), respectively. These camera-lucida montages are selective tracings of mushroom body extrinsic neurons. $E-H$ therefore appear different from montages of intrinsic neurons from the same strains published in Ito et al. (1997a). $(A, B)$ The flp clone (diagram in Fig. 5G) has a cluster of cell bodies in the dorsolateral region of the anterior cortex (arrowheads). The cells innervate bilaterally the $\gamma$ lobe, three regions of the anteriormost inferior medial protocerebrum (medial, lateral 1, and lateral 2 of the i $\mathrm{m} \mathrm{pr}$ ), and ventral bodies $(\mathrm{v} \mathrm{b})$. The roots of the $\alpha$ lobes are also invaded, though sparsely. The fibers cross the midline at the posterior region of the $\gamma$ lobe $(\rightarrow) .(C, D)$ GAL4 enhancer-trap strain Mz 19 labels a subset of neurons labeled in the flp clone (arrowheads; diagram in Fig. $5 \mathrm{H}$ ). N ote that, unlike the flp clone, the enhancer trap system labels corresponding cells in the cortex of both sides of the brain. These neurons arborize in the $\gamma$ lobe and the medial and lateral 1 regions of the i $\mathrm{m}$ pr. Presynaptic sites were found in the $\gamma$ lobe and the lateral 1 regions (see Fig. 5B). (E,F) Line Mz 717 shows apparently ubiquitous staining of the mushroom bodies. This, however, is caused by the labeling of only a small subset of Kenyon cells, which are dispersed in the pedunculus (ped) and lobes. In addition, a pair of interior neurons labeled (diagram in Fig. 5I), with large cell bodies in the posterior cortex (arrowheads), crosses the neuropil and bifurcates (black arrows) near the lateral edge of the fan-shaped body ( $\mathrm{b}$ ). Their medial branches invade the head of the $\gamma$ lobe. The lateral branches reach the shaft of the vertical lobe and further bifurcate (white arrow) to project to the spur (sp) and to the head of the $\alpha^{\prime}$ lobe. The arborization areas of these neurons are, however, restricted to within the mushroom bodies. $(G, H)$ Line $N p 100$ labels a different small subset of Kenyon cells and a pair of extrinsic neurons that arborize only in the contralateral brain half (diagram in Fig. 5J, probably same cell as in Fig. 3B, left). The cell body (arrowhead) lies in the anterior cortex, lateral to the spur. The fiber passes beneath the pedunculus, crosses the midline in front of the ellipsoid body (e b) and bifurcates behind the head of $\gamma$ (arrow). Its ventral branch invades the $\beta$ head, whereas the dorsal branch climbs through the i $\mathrm{m}$ pr and arborizes at the $\alpha$ lobe head and the $\mathrm{s} \mathrm{m}$ pr around it. As shown in Fig. $5 \mathrm{~F}$, arborizations of this cell type contain presynaptic sites both in the i $\mathrm{m} \mathrm{pr}$ and $\mathrm{s} \mathrm{m} \mathrm{pr}$. Scale $=50 \mu \mathrm{m}$.

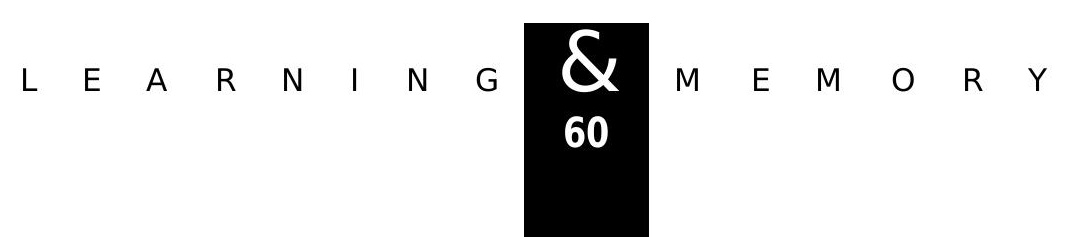




\section{MUSHROOM BODY EXTRINSIC NEURONS}

Dickson, unpubl.) were used in this study to reveal the whole cellular structures. Unlike Golgi preparations, the stained fibers can always be traced back to the cell bodies.

In contrast to the obvious polarity of sensory and motor neurons, the direction of signals within a netw ork of interneurons is difficult to define. For this purpose, it is important to distinguish within a neuron its presynaptic (output) and postsynaptic (input) specializations. Morphologic criteria, as suggested in the next section, are at best unreliable, whereas electron microscopic examination of each cell would be impractical. To address this problem, we developed a new reporter construct,
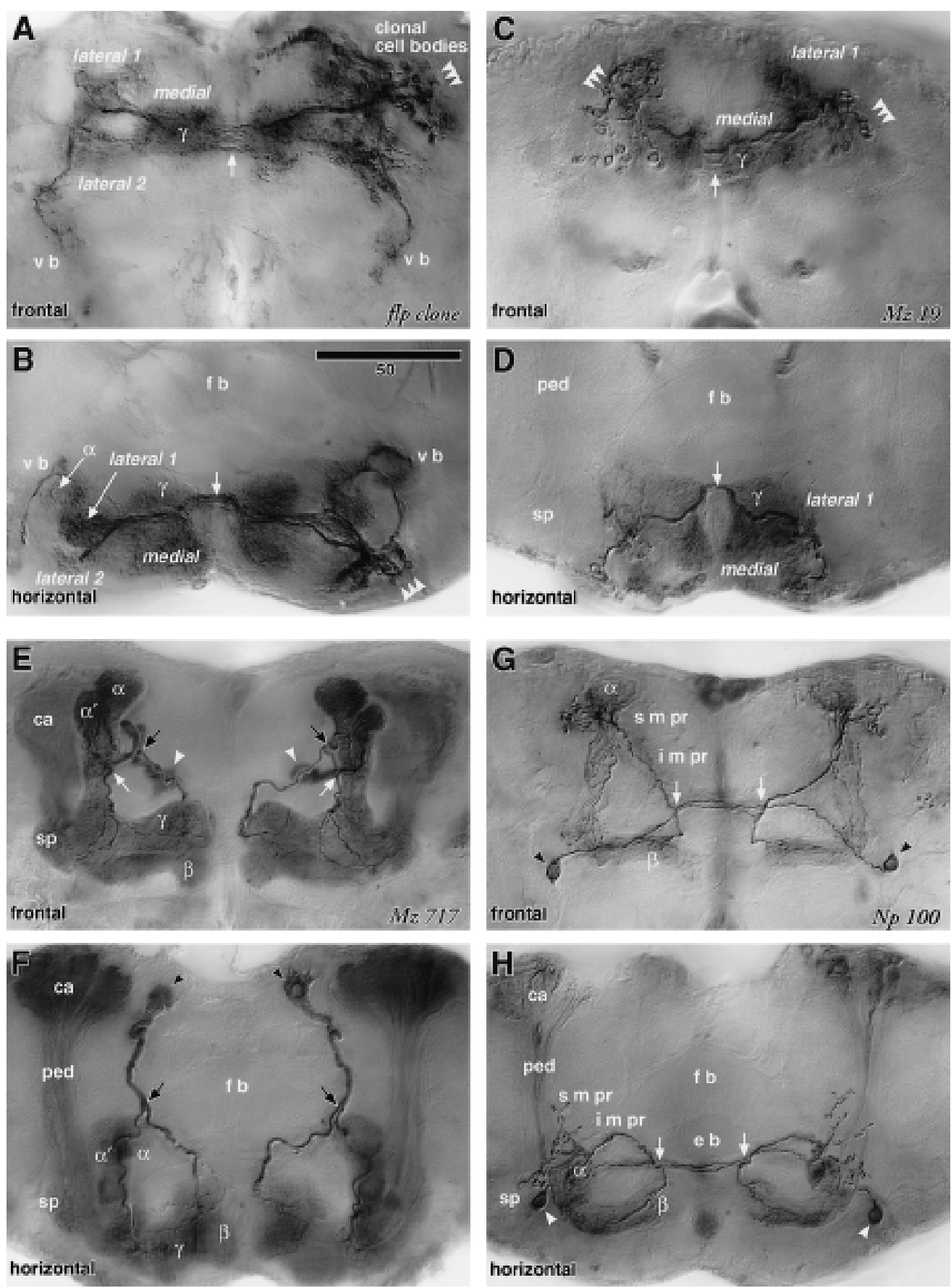

Figure 4: (See facing page for legend.)

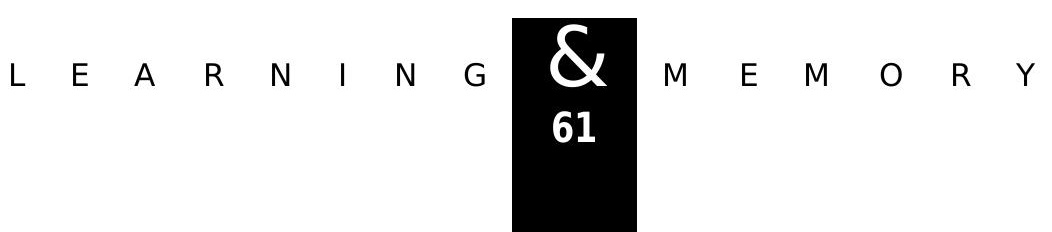


Ito et al.

UAS-n-syb-GFP, that provides a reliable indication of what parts of a neuron are predominately donative in function. With the presynaptic vesicle-specific neuronal synaptobrevin protein fused to GFP, the reporter is transported actively to those parts of a neuron's processes that contain presynaptic sites (P.E. Estes, G. Ho, D. Hurd, W. Saxton, and M. Ramaswami, in prep.).

By using high-power Nomarski interference optics, it was possible to recognize the outlines of the mushroom bodies even if the region is not itself labeled. This enabled us to determine whether taulabeled fibers are indeed associated with the mushroom bodies. In confocal microscopy, background fluorescence reveals the boundary of mushroom bodies in each optical section, making it possible to determine the association of the GFP-and n-sybGFP-labeled fibers with the mushroom body neuropils.

The bulbous swellings at the head of the $\gamma$ lobe and the surrounding i $\mathrm{m} \mathrm{pr}$ are interconnected by a group of extrinsic neurons that also link the heterolateral lobes (Figs. 4, C and D, and 5, A and H). The presynaptic sites associated with these neurons exist both within and outside the $\gamma$ lobe (Fig. $5 B)$. This suggests that the information flow between the $\gamma$ lobe and the i $\mathrm{m} \mathrm{pr}$ is bidirectional. The flp clone show s that these neurons are clonally related (Figs. 4, A and B, and $5 \mathrm{G}$ ). The same clone also contains neurons that link the $\gamma$ lobe with more lateral areas of the i $\mathrm{m} \mathrm{pr}$ as well as the ventral body.
The head and root (i.e., its origin from the pedunculus) of the $\gamma$ lobe have distinct morphological characteristics. An interior neuron (Figs. 4, $E$ and $F$, and $5, C, D$, and I) sends two branches to the $\gamma$ lobe. The component supplying the head of the $\gamma$ lobe arises separately from the other branch that invades both the $\gamma$ lobe root and the head of the $\alpha^{\prime}$ lobe. The neuron labeled 1 in Figures 6, E and $F$ (and Fig. 7l) also forms separate arborizations in the head and root of the $\gamma$ lobe. The arborizations of the observed extrinsic neurons are limited to within the lobes and do not invade the pedunculus.

The projections to the superior protocerebrum are associated closely with the head of the $\alpha$ lobe. Although the neuron in Figures $4, \mathrm{G}$ and $\mathrm{H}$, and $5, E, F$, and $J$ has arborizations in the head regions of both the $\alpha$ and $\beta$ lobes, projections to the surrounding neuropil ( $\mathrm{s} \mathrm{m} \mathrm{pr}$ and i $\mathrm{m} \mathrm{pr}$ ) are limited to the branch towards the $\alpha$ lobe. Two neurons are found to link the lateral horn and the head of $\alpha$ (Figs. 6, A-D, and 7, A-D, G, and H). In both cases the $\mathrm{s} \mathrm{m}$ pr is invaded together with the $\alpha$ lobe. Neurons with extensive arborizations in the $\mathrm{s} I \mathrm{pr}$ and $\mathrm{s} \mathrm{m}$ pr also contact the $\alpha$ lobe (Figs. $6, G$ and $H$, and $7, E, F$, and $J$ ).

Consistent with observations of Golgi and Bodian preparations, GAL4-based labeling show s that, outside the mushroom bodies, extrinsic neuron processes invade delineated neuropils: an anterior area of the i $\mathrm{m}$ pr partially encircling the medial lobe and situated behind the shaft of the vertical

Figure 5: Stereographs and diagrams of the neurons shown in Fig. 4. $(A-F)$ Stereographs of three dimensional reconstruction (frontal view) showing cytoplasmic $\operatorname{GFP}(A, C, E)$ and presynaptic $n$-syb-GFP $(B, D, F)$ labeling of enhancer trap strains. Unlike digital camera-lucida montages, confocal reconstruction records all labeled structures. $(A)$ Line $M z 19$ labels iACT neurons between the antennal lobe (ant lob) and lateral horn (I ho), and mushroom body extrinsic neurons between the $\gamma$ lobe and i $\mathrm{m}$ pr (diagram in $H$, see also Fig. 4C,D). The latter is the subset of neurons labeled in the flp clone (diagram in $G$, see also Fig. 4A,B). Cytoplasmic GFP reveals all fiber structures. (B) Presynaptic $n$-syb-GFP reveals presynaptic sites both in the $\gamma$ lobe and i m pr. iACT neurons reveal presynaptic sites at both ends of the tract (ant lob, $c a$, I ho). (C) GFP labeling of the small subset of Kenyon cells labeled in line Mz 717 reveals the tightly bound $\alpha$ and $\beta$ lobes, and the looser fibers of the $\alpha^{\prime}$ and $\gamma$ lobes and spur (sp). A pair of interior neurons (diagram in I, see also Fig. 4E,F) projects from the posterior cortex to the lobes. (Note that fibers from other labeled cells lie above or beneath this neuron). (D) $n$-syb-GFP staining reveals that these subsets of Kenyon cells carry presynaptic cites only in the lobes and spur, but not in the calyx and pedunculus (ped). The presynaptic regions of the interior neurons cannot be recognized because of the staining of these Kenyon cells. $(E)$ Line Np 100 shows the projection of a pair of extrinsic neurons with cell bodies just below the spur (sp, diagram in J, see also Fig. 4G,H). Label is also seen in a few Kenyon cells that project only to the $\alpha$ and $\beta$ lobes, as well as in the median bundle ( $\mathrm{m} \mathrm{bdl}$ ) cells and several cells in lateral neuropils. $(F)$ The extrinsic neurons carry presynaptic sites in their projections to the i $\mathrm{m}$ pr and $\mathrm{sm} \mathrm{pr}$, behind the $\alpha$ lobe. Because of the staining of the Kenyon cells, it is not clear whether these extrinsic neurons have presynaptic sites also within the lobes. (G-J) Diagrams of extrinsic neurons described in Fig. 4 and here (oblique frontal view from the anterior). (O) Labeled cell bodies; (shaded ellipsoids) primary arborizations; (black ellipsoids) presynaptic sites. (K) Control staining of the newly developed UAS- $n$-syb-GFP strain. W ithout GAL4 driver, the line shows no labeling in the neuropil. The occasional random dots in the cortex can be disregarded as noise (see Material and Methods).

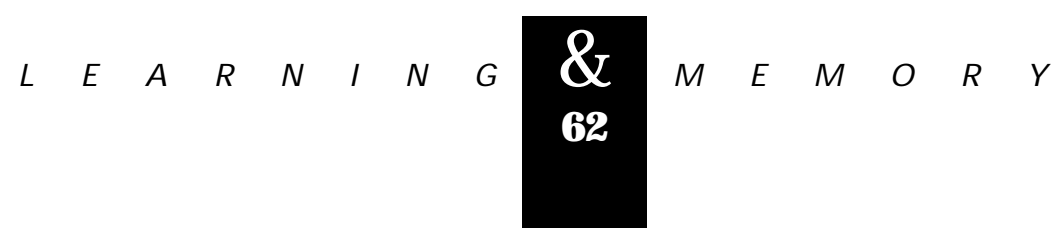




\section{MUSHROOM BODY EXTRINSIC NEURONS}

lobe, and anterior neuropils of the $\mathrm{s} \mathrm{m} \mathrm{pr}$ and $\mathrm{s} / \mathrm{pr}$, located near the head of the vertical lobe. Except for tw 0 neurons that link the $\alpha$ lobe with the lateral horn, no projections were observed to reach from the mushroom bodies to other parts of the brain, such as the inferior lateral protocerebrum (i I pr) and the posterior brain including the posterior slope. The flp clone is so far the only example that reveals the connection between the mushroom body lobes and the ventral body. No projections were found to form direct connections between the mushroom body and the central com-
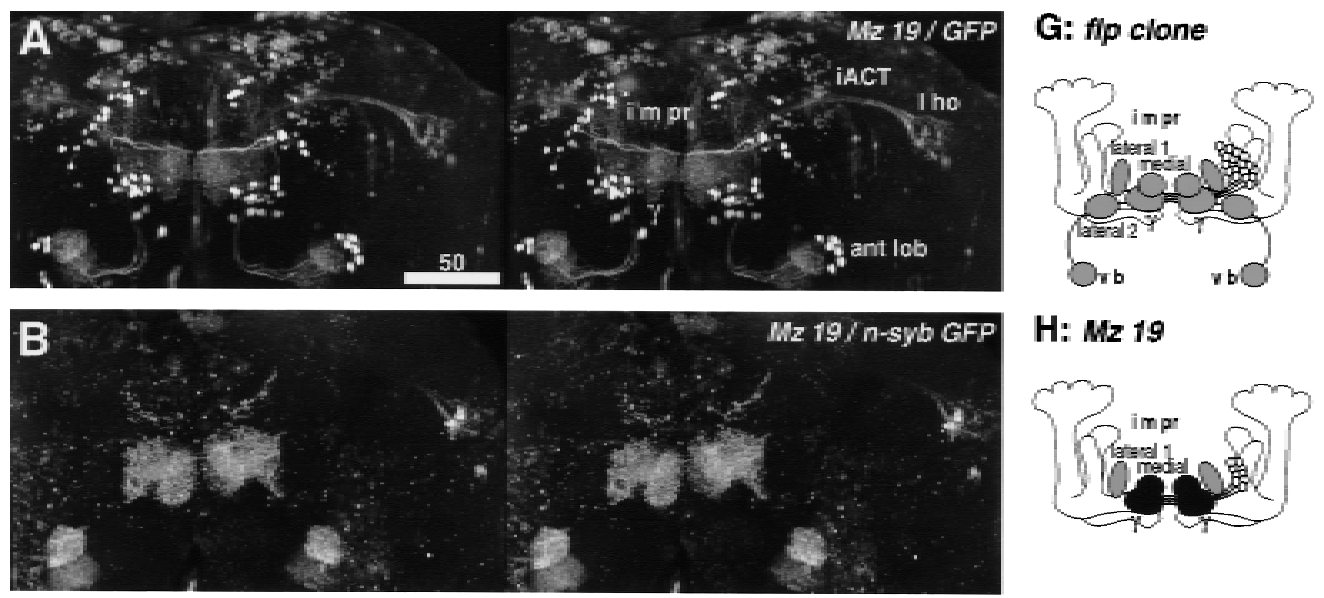

\section{H: Mz 19}
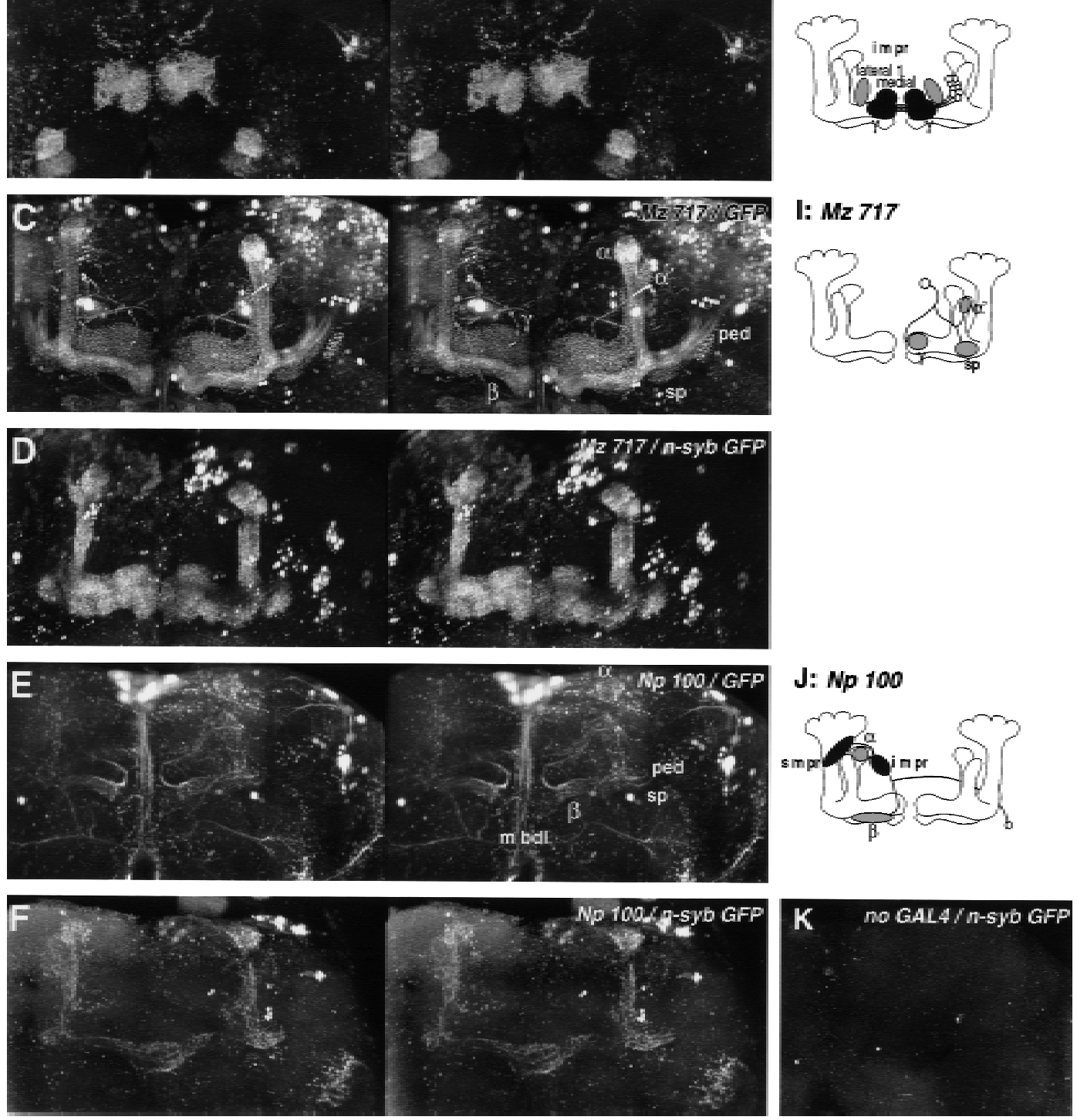

Figure 5: (See facing page for legend.)

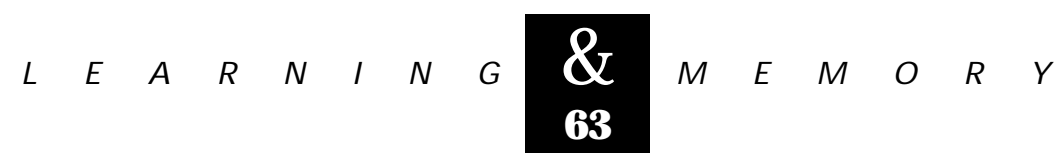


Ito et al.

plex, which is suggested to have higher-order motor-associative functions (Strauss and Heisenberg 1993).

As show $n$ by the flippase clone and the GAL4 strain Mz19 (Figs. 4 and 5), certain types of mediallobe extrinsic neurons exist as clusters. How ever, so far, such clusters have not been found around the vertical lobe. This would explain why Bodian staining reveals more fibers in the medial lobe, and why extrinsic neurons associated with the medial lobes are observed more frequently amongst Golgi preparations (see previous section).
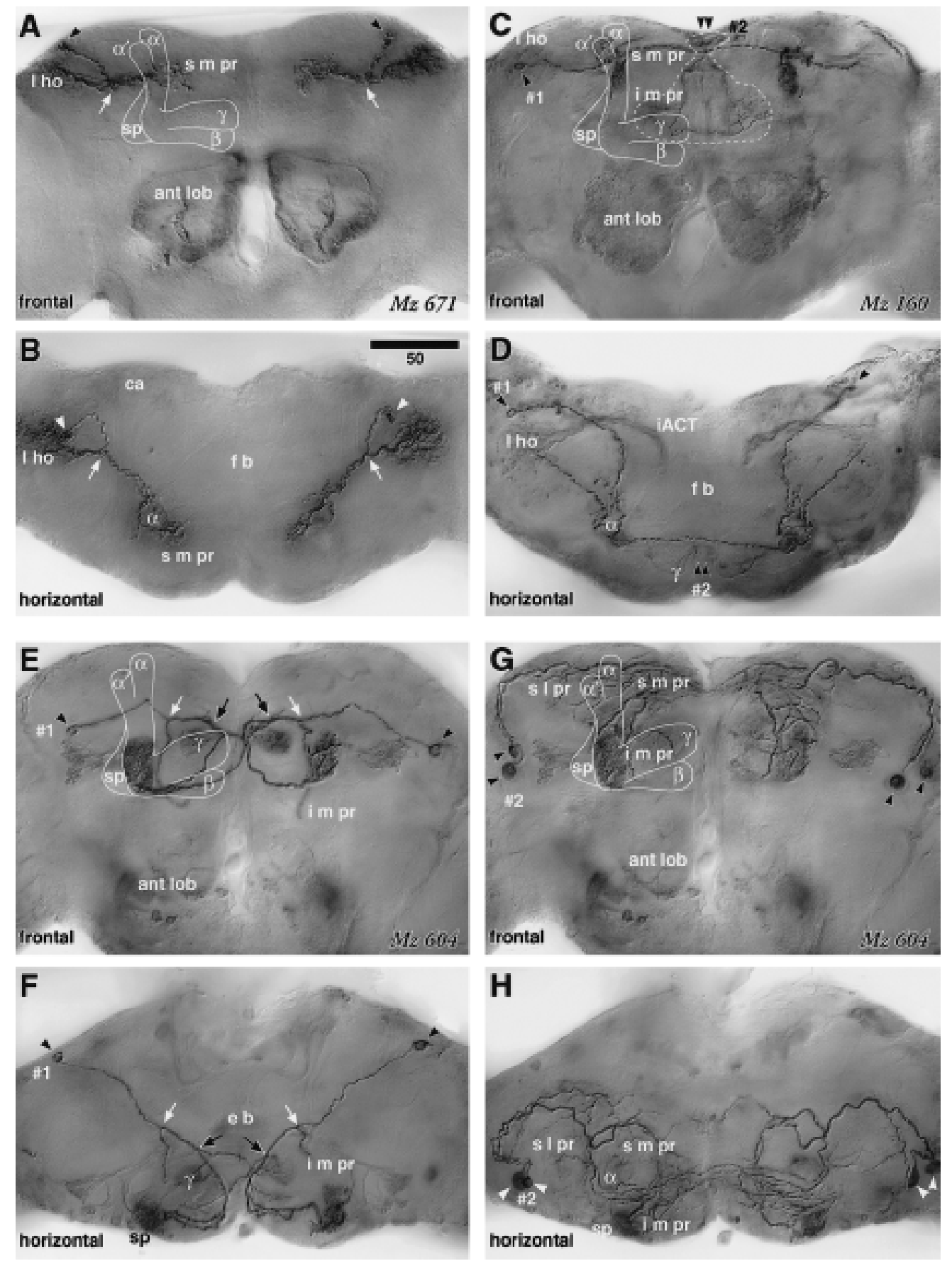

Figure 6: (See facing page for legend.)

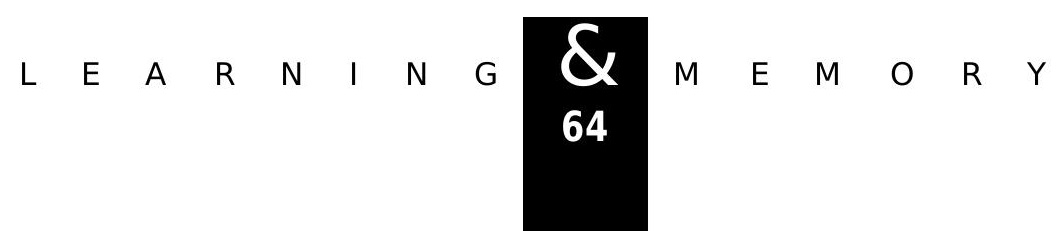




\section{CELL TYPES OF THE MUSHROOM BODIES}

The present data allow the identification of the following six cell types associated with the mushroom bodies of Drosophila.

\section{ASCENDING SENSORY AFFERENTS}

Ascending sensory afferents are the projections to the calyx region. In Drosophila, only those from the olfactory system have been documented so far. They comprise projection neurons (relay interneurons) of the inner antennocerebral tract (iACT; Figs. 1 and $3 \mathrm{~A}$, right). It is likely that in Drosophila, as in other species (e.g., Manduca sexta; Tolbert and Hildebrand 1981), the dendrites of projection neurons are postsynaptic to local interneurons and to olfactory receptor afferents in the antennal lobe glomeruli. A small subset of the antennal lobe neurons in the middle antennocerebral tract (mACT) are observed to enter the pedunculus and terminate at the calyx (Stocker et al. 1990). Although functional evidence is still lacking in flies, the antennal lobe relay interneurons of locusts and honeybees have been shown to carry specific information about odor types processed in the antennal lobes (Stopfer et al. 1997). The number of Drosophila iACT afferents is <200, many few er than the $\sim 3000$ Kenyon cells that are postsynaptic to them (Stocker et al. 1990). As dia- grammed in Figures 1 and $3 \mathrm{~A}$ (right), iACT neurons terminate eventually in the lateral horn, where they converge with other olfactory interneurons from the main trajectory of the MACT (Fig. 1). This arrangement of terminals, which is common to other insect groups (Homberg et al. 1989), demonstrates that the lateral horn, rather than the mushroom body, contains the largest number of olfactory interneuron terminals.

\section{INTRINSIC NEURONS}

Intrinsic neurons, first identified in honeybees by Kenyon (1896) and hence called Kenyon cells (Strausfeld 1976), can be subdivided into several morphological types according to their dendritic morphology (Mobbs 1982), and biochemical types as suggested by enhancer activity (Yang et al. 1995; Ito et al. 1997a). Two morphologies have been identified in Drosophila. The first (Fig. 3A, left) has dendrites in the calyx equipped with claw -like specializations that clasp the swollen varicosities arising from the axon collaterals of IACT neurons (Fig. $3 \mathrm{~A}$, right), and send axonal branches through the $\gamma$ and $\alpha^{\prime}$ lobes. The other type of Kenyon cell has delicate pinnate dendrites. These Kenyon cells send slender axon branches through the $\beta$ and $\alpha$ lobes (Fig. 3B, right, and 4, G and H). A distinguishing feature of these two cell types is that the former provides axons that have convoluted trajec-

Figure 6: Extrinsic neurons connecting the mushroom body and lateral protocerebrum. $A-D$ are digital camera-lucida montages of identical preparations viewed frontally (dorsal to the top) and horizontally (anterior to the bottom). $E-H$ are each montages of the same preparation but tracing different neurons (1 and 2). (A,B) GAL4 enhancer-trap strain Mz 671 labels a pair of extrinsic neurons that connect the lateral horn (I ho) and the $\alpha$ lobe (diagram in Fig. 7G). The cell body lies in the posterior dorsal cortex (arrowhead), and the fiber bifurcates in the dorsal protocerebrum (arrow). Its lateral branch provides extensive arborizations in the lateral horn. The medial branch crosses the neuropil obliquely, circumvents the shaft of the $\alpha$ lobe, and arborizes around the shaft in the superior medial protocerebrum (s $\mathrm{m}$ pr). Some arborizations also invade the head of the $\alpha$ lobe. The neuron has presynaptic sites in the $\mathrm{s} \mathrm{m} \mathrm{pr}$ and lateral horn, but not in the $\alpha$ lobe head (see Fig. 7B). $(C, D)$ Cell type (1) labeled by $M z$ 160, has its cell body in the posterior cortex (arrowhead, diagram in Fig. $7 \mathrm{H}$ ). Its cell body fiber runs across the dorsal neuropil to project to the $\alpha$ lobe, where it forms an elaborate arborization in which n-syb GFP-staining demonstrates presynaptic sites (see Fig. 7D). The nearby s $\mathrm{m}$ pr is also invaded by processes, and a lateral branch projects to the lateral horn (I ho). Another branch projects to the contralateral $\alpha$ lobe head. (2) Cell type with cell bodies in the dorsal midline cortex (arrowhead) that sends fibers to the i $\mathrm{m}$ pr just above the $\gamma$ lobes, but does not invade the mushroom body. $(E, F) O$ ne of the cells labeled in $M z 604(1)$ has its cell body in the posterior cortex (arrowheads) and projects through the inferior protocerebrum (diagram in Fig. 7l). It forms its first bifurcation (white arrow) lateral to the ellipsoid body (e b), sending a branch ventrally to the i $\mathrm{m}$ pr just above the ant lob. This is succeeded by $a$ second bifurcation (black arrow) from which a branch crosses the midline and projects to the contralateral mushroom body's spur (sp). The ipsilateral branch forms a small arborization in the posterior swelling of the $\gamma$ lobe head, extends further in front of the $\gamma$ lobe, and follows the contralateral projection of its opposite counterpart to form an extensive arborization within the root of the $\gamma$ lobe, which contains numerous presynaptic sites (Fig. 7F). (G,H) Labeled cell type (2) that derives from two cell bodies in the anterior lateral cortex (arrow heads, diagram in Fig. 7J). The cell body fibers project along the perimeter of the $\mathrm{s} I \mathrm{pr}$, where they provide extensive branches along the surface of the $\mathrm{s} I \mathrm{pr}, \mathrm{s} \mathrm{m} \mathrm{pr}$, and i $\mathrm{m}$ $\mathrm{pr}$, and also invade the $\alpha$ lobe and the spur (sp). Scale $=50 \mu \mathrm{m}$.

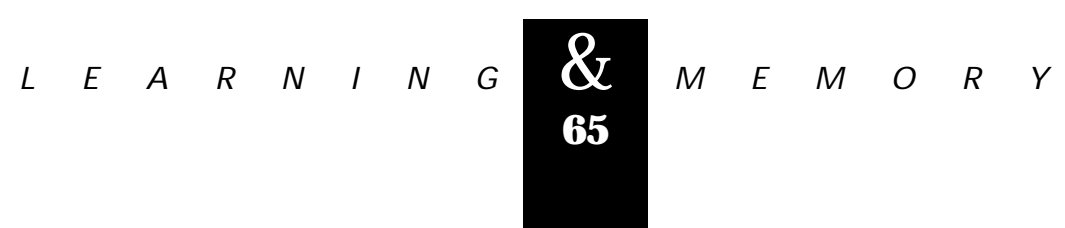


Ito et al.

tories in the $\gamma$ lobe, sometimes branching there and providing whorls of processes that contribute to the terminal swellings of $\gamma$, whereas the latter have thinner, straighter trajectories and are bound more tightly. Both types of cells are found in each of the four clonally distinct cell groups (Ito et al. 1997a). The thinner fibers from the four clones form four bundles in the core of the posterior pedunculus, which coalesce at the anterior end of the pedunculus and continue into $\beta$ and $\alpha$ (Fig. 1).

\section{INTERIOR NEURONS}

Interior neurons are intrinsic in that their synaptic processes are limited to within the mushroom body lobes (Figs. $3 E$ and $4 \mathrm{E}$ and $\mathrm{F}$ ), but would be classified as extrinsic after Mobbs' (1982) criteria, since they are derived from protocerebral cell bodies that lie distant from the mushroom bodies. Their designation "interior" is given to avoid confusion with the intrinsic Kenyon cells.

\section{MUSHROOM BODY LOBE EFFERENTS}

Mushroom body lobe efferents, or output neurons, are a class of extrinsic neurons that are genuine relay neurons having axons that extend from the lobes to other brain areas [Figs. 3, B (left), C (left), D (left), D (right), and $G$ (right), and $4, C$ and $D, G$ and $H$, and $6, A$ and $B, E$ and $F$ ]. Their processes in the lobes are equipped with spines or minute pin-head like structures (Fig. $2 F$ and $G$ ) that, with reference to electron microscopic studies on other Drosophila neurons (Meinertzhagen and O'Neil 1991) are interpreted as postsynaptic specializations. GAL4-responsive n-syb-GFP expression in these neurons reveals the site of presynaptic vesicles, and presumably presynaptic sites, in the regions outside the lobes (Figs. 5, B and $F$, and $7, B$ and F). Efferent neurons send axons to regions of the protocerebrum where they terminate as varicose arbors. Certain efferent neurons provide connections both to within other parts of the mushroom body and to regions outside it. These neurons have dendrite-like arborizations in the medial lobes and branches that extend to the heads of vertical lobes as well as to the $\mathrm{s} m \mathrm{pr}$ outside [Figs. 3, B (left) and C (left), and 4, G and H].

\section{MUSHROOM BODY LOBE AFFERENTS}

Mushroom body lobe afferents are defined as neurons that supply the lobes from dendrites in the protocerebrum (Figs. 2, D and E, 3F, 4, A-D, and 6, $\mathrm{C}-\mathrm{F})$. Their terminals in the lobes are varicose or blebbed, and correspond to presynaptic specializations. Comparable neurons have been identified in the cockroach Periplaneta americana, in which they have been shown to carry multimodal information (Li and Strausfeld 1997a,b). The distinction between mushroom body lobe efferents and afferents can be ambiguous, however, because certain extrinsic neurons have presynaptic vesicles, and presumably presynaptic sites, both within the lobes and the surrounding protocerebra (Figs. 5B, and $7, \mathrm{D}$ and $\mathrm{F}$ ). The fact that these cells suggest bidirectional polarity is not surprising, as other studies have shown that the terminals of insect neurons, as well as their dendritic trees, can simul-

Figure 7: Stereographs and diagrams of the neurons shown in Fig. 6. $A-F$ are three dimensional stereographs of reconstructions (frontal view) of cytoplasmic GFP $(A, C, E)$ and presynaptic $n-\operatorname{syb}-G F P(B, D, F)$ labeling. (A) GFP staining of the line $M z 671$ reveals the connection between the lateral horn (I ho) and the $\alpha$ lobe head as well as the surrounding $\mathrm{s} \mathrm{m}$ pr (diagram in $G$, see also Fig. 6A,B). (B) Presynaptic sites are found in the $s \mathrm{~m} \mathrm{pr}$, but not in the $\alpha$ lobe (confirmed by examining each confocal section). Very faint staining is also observed in the lateral horn. (C) $M z 160$ labels another connection between the lateral horn (I ho) and the $\alpha$ lobe/s $\mathrm{m}$ pr (diagram in $H$, see also Fig. 6C,D). Also labeled are iACT neurons with arborizations in the antennal lobe (ant lob), calyx (ca), and lateral horn. 0 ther labeling is in projections to the lateral triangle (I tr) of the central complex, commissures between the ventrolateral protocerebra ( $\mathrm{I}$ pr), and tracts connecting the $\mathrm{v} I \mathrm{pr}$ and lateral horn. $(D)$ U nlike $M z 671$, the $\alpha$ lobe head of this extrinsic neuron possesses presynaptic sites (confirmed by examining each confocal section), suggesting that the $\alpha$ lobe receives afferents. Because of the staining of the iACT terminals, we could not confirm whether this neuron also has presynaptic sites in the lateral horn. (E) Line $M z$ 604 labels two types of extrinsic neurons ( 1 and 2, diagram in $I$ and $J$, see also Fig. $6 \mathrm{E}-\mathrm{H}$ ). Also labeled are commissures (co) connecting antennal mechanosensory regions, as well as projections from cells in the posterior cortex to the fanshaped body ( $(\mathrm{b})$, ellipsoid body (e b), noduli (no), and from cells in the anterior cortex to the lateral triangle (I tr). $(F)$ Presynaptic sites of neuron 1 are found in the root of the $\gamma$ lobe and in the i $\mathrm{m} \mathrm{pr}$. Those of neuron 2 can be seen in the $\mathrm{s} \mathrm{m}$ pr near the midline and in the $\mathrm{s} \mathrm{m}$ pr in front of the shaft of the vertical lobe. $\mathrm{G}-\mathrm{J}$ are diagrams of extrinsic neurons described in Figs. 6 and 7. Labels as in Fig. 5. The white ellipsoid denotes the area confirmed to be free from presynaptic sites.

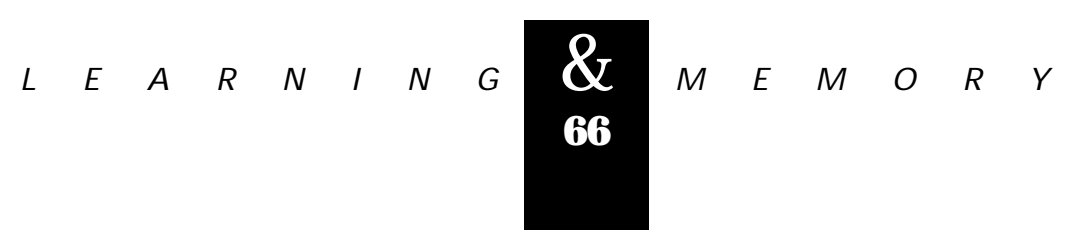




\section{MUSHROOM BODY EXTRINSIC NEURONS}

taneously be pre- and postsynaptic (for review, see Strausfeld and Meinertzhagen 1998). What defines a terminal is not its distance from its cell body but its preponderance of presynaptic sites.

\section{GLIAL CELLS}

Glial cells are also found in the mushroom body structure. Neuropil-associated glial cells (Ito et al. 1995) have multipolar cell bodies within the neuropil (Figs. 2B and $3 \mathrm{C}$, right). They provide velate processes that occupy zones within the lobes that correspond to the dendritic domains of specific extrinsic neurons. Other neuropil-associated glial cells separate bundles of axons in the calyx,
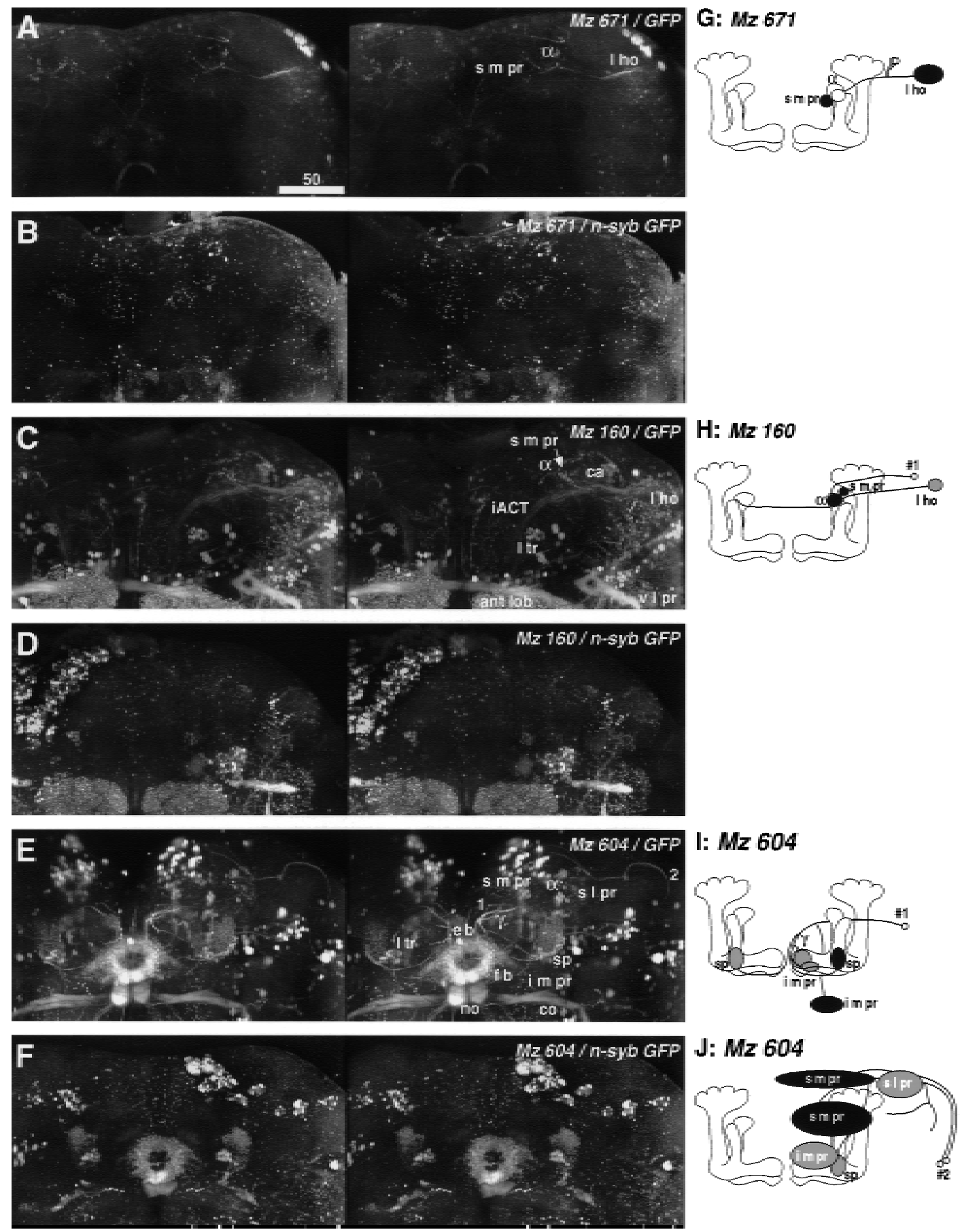

Figure 7. (See facing page for legend.)

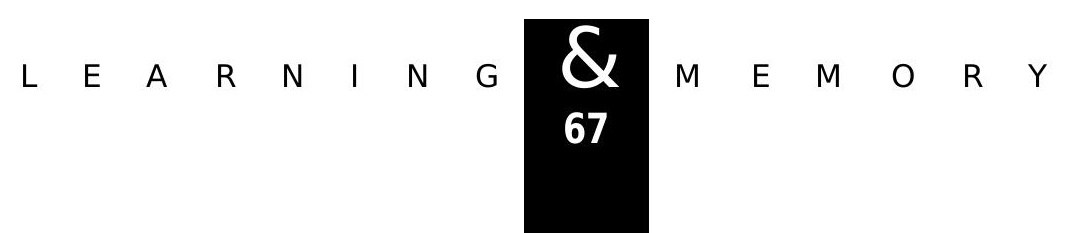


Ito et al.

and cortex-associated glial cells lie among the Kenyon cell bodies (see Fig. 5 in Ito et al. 1997a).

The majority of the cell bodies of categories 3-5 are located in the anterior cortex. A few of them, how ever, have their cell bodies in the lateral or posterior cortex, linked by a long cell-body fiber to its arborizations in the lobes (Figs. 4F, and 6, B, $D, F$, and $H$ ).

\section{Discussion}

This study is the first systematic attempt to identify the neuronal connections between the mushroom body lobes and other neuropils. Compared to the total number of Golgi-impregnated preparations, as well as the number of GAL4 enhancer-trap strains and flippase clones, the inci- dence of staining or labeling mushroom body extrinsic neurons was small. In contrast, other types of neurons, such as Kenyon cells and optic lobe neurons, were frequently and abundantly identified. For example, screening of the 500 GAL4 enhancer-trap strains gave only six strains that label mushroom body extrinsic neurons but $>50$ strains that label various subsets of Kenyon cells. This suggests that the total number of mushroom body extrinsic neurons is very low indeed and disproportionately smaller than the number of Kenyon cells ( 3000 per hemisphere).

Some cell types described in this study were observed in only a single preparation or single strain, suggesting that further screening might reveal a few additional extrinsic neurons. How ever, the two fundamentally different methods, Golgi-

Figure 8: Schematic of information flow to and from the Drosophila mushroom body. Olfactory and gustatory stimuli are processed in the antennal lobe and the gustatory center of the subesophageal ganglion (sog), respectively. The feedback loop (behavior) indicates that motor actions feed back as new sensory stimuli. Projections from the antennal lobe convey information to the lateral horn (via iACT and mACT) and to the mushroom body (predominantly via iACT, with a few fibers via $m A C T)$. Projections via the $O A C T$ and broad root (BR) send information to the inferior lateral/medial protocerebra. Projections from the gustatory center join the iACT (Ito, unpubl.). Kenyon cells of the mushroom body supply thick axons to $\gamma$ and $\alpha^{\prime}$ lobes, and slender axons to $\beta$ and $\alpha$. The two types of Kenyon cells are biochemically heterogeneous (represented by different shades). Extrinsic neurons form presynaptic (small circles) or postsynaptic (small rectangles) connections with the Kenyon cell fibers. The direction of information is show $n$ with a small arrowhead. Note that each extrinsic neuron does not necessarily interact with all the Kenyon cell fibers, and the strength of each connection is unlikely to be the same. Efferent and afferent neurons ( $1-4)$ connect the medial $(\beta$ and $\gamma$ ) lobes with surrounding mushroom body-linked neuropils; e.g., the

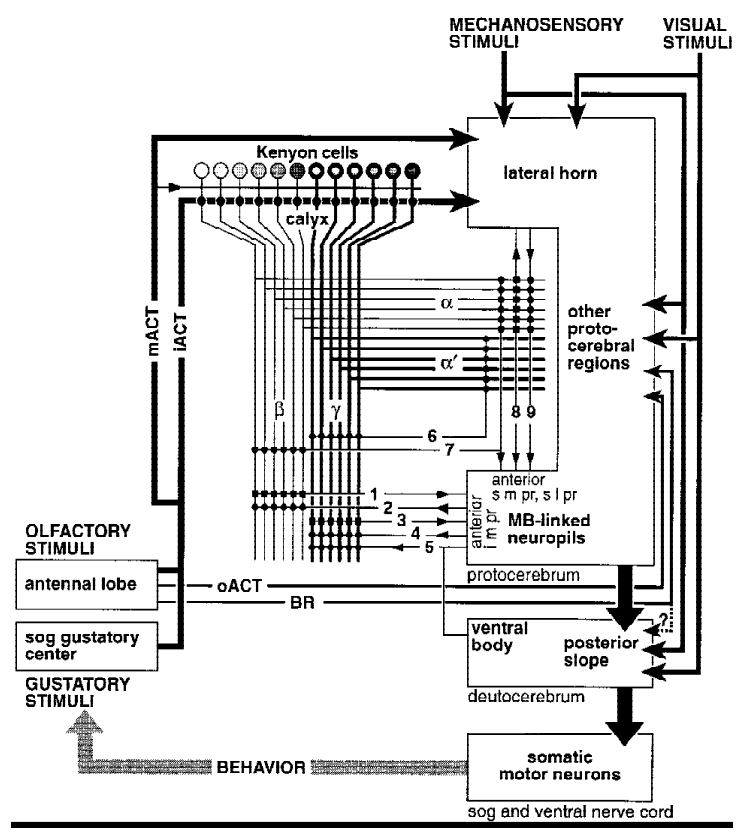
anterior portion of the i $\mathrm{m}$ pr. Input to the medial lobes is also likely to be provided from the ventral body (5), although the direction of signals is yet unknown. Interior neurons (6) connect, or are common to, the vertical ( $\alpha$ and $\left.\alpha^{\prime}\right)$ and medial lobes. Relatively few extrinsic neurons link vertical lobes to the surrounding mushroom body-linked neuropils (anterior portion of the $\mathrm{s} \mathrm{m}$ pr and $\mathrm{s} / \mathrm{pr}$ ). Some of them (7) connect both vertical and medial lobes with the surrounding neuropil. A few neurons $(8,9)$ connect the lateral horn and the head of the $\alpha$ lobe; these neurons also have projections in the $s \mathrm{~m}$ pr near the lobe head. Mechanosensory and visual information is sent to the lateral horn and other protocerebral regions, from where signals might be relayed to the mushroom body-linked neuropils and then to the mushroom body lobes. Actual projections between these areas remain unknown. These mechanosensory and visual neuropils also have direct connections to the posterior slope, which contains premotor neuropil supplying descending neurons to the subesophageal ganglion and thoracic-abdominal ganglia (ventral nerve cord). Although suggested (dotted line), direct projections from the ant lob via the broad root (BR: Pow er 1946) to the posterior slope have not been observed. The posterior slope receives fibers from protocerebral neuropils (thick arrow), but little is known about their origin. Connections within and between protocerebral neuropils are left essentially uninvestigated (within area designated as containing mushroom body-linked neuropils, lateral horn, and other protocerebral regions). Information from the mushroom bodies is sent to these neuropils, rather than directly to the posterior slope.

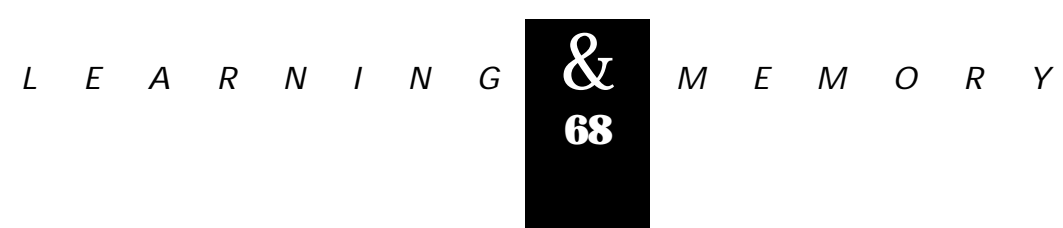


and GAL4-based labeling, reveal cellular structures that are highly comparable. Certain of these cell types appeared more than once within the library of Golgi preparations, suggesting that we have found a significant portion, if not all, of the entire population of the extrinsic neurons (schematized in Fig. 8).

Based on our observations, we discuss structural heterogeneity in the internal organization of the mushroom bodies, protocerebral neuropils that are connected with them, and the functional interpretation of these anatomical arrangements.

\section{DIFFERENCES BETWEEN THE PEDUNCULUS AND THE MEDIAL AND VERTICAL LOBES}

We found that the pedunculus and the vertical and medial lobes of the Drosophila mushroom bodies have distinct characteristics, even though they are essentially supplied by the fibers of the same intrinsic neurons. The pedunculus contains few if any extrinsic neurons. Although the mushroom bodies penetrate the entire protocerebrum from posterior to anterior, interaction with other neuropils is limited to their calyces and lobes.

The vertical lobes have significantly few er extrinsic elements than the medial lobes. Whereas extrinsic neuron dendrites are located throughout the medial lobes, extrinsic neuron processes mainly invade the head of the vertical lobes, but not their shafts. The projection patterns of extrinsic neurons suggest that the medial and vertical lobes interact with different protocerebral regions. The medial lobes are connected with the anterior part of the i $\mathrm{m} \mathrm{pr}$, whereas the heads of the vertical lobes are connected to the $\mathrm{s} \mathrm{m} \mathrm{pr}$ and $\mathrm{s} / \mathrm{pr}$. Thus, the medial and vertical lobes are associated closely with the neuropils that immediately surround them. The only exception identified so far is the connection between the vertical lobe and the lateral horn.

Evidence from other taxa also suggests that the vertical and medial lobes differ significantly. In honeybees, focal cooling of the antennal lobe and the nearby $\alpha$ lobe, but not the $\beta$ lobe, perturbs the acquisition of short-term olfactory memory (Erber et al. 1980). Disruption of the $\beta$ lobe, but not the $\alpha$ lobe, abolishes maze learning in the ant Formica rufus (Vowles 1964). Similarly, place memory of cockroaches is perturbed by bilateral lesions of the pedunculus and $\beta$ lobes, but not the $\alpha$ lobes (Mizunami et al. 1993). Bodian staining of the cock- roach mushroom bodies suggests that the $\alpha$ and $\beta$ lobes have distinct cytoarchitectures, and intracellular dye fills show the vertical and medial lobes to provide different classes of efferent neurons ( $\mathrm{Li}$ and Strausfeld 1997a). In locusts, staining for nitric oxide synthase reveals longitudinal subdivisions of the $\alpha$ lobe but not in $\beta$ (Elphick et al. 1995; Bicker and Hähnlein 1995).

\section{INTERNAL SUBDIVISIONS WITHIN THE LOBES}

Extrinsic neurons occupy characteristic positions within each lobe. The heads of the vertical lobes receive a greater abundance of extrinsic neuron processes than do their shafts. In the medial lobes, the terminal swellings and the root of the same lobe are often invaded by separate arborizations of a single extrinsic neuron (Figs. 3E, 4, E and $F$, and $6, E$ and $F$ ). Heterolateral connections that link the corresponding lobes in each hemisphere are limited to the terminal sw ellings of the lobes. A second type of division is suggested by the transverse subdivisions of the lobes by the invading glial cell processes. Their domains possibly serve to isolate discrete regions of synaptic connections among Kenyon cells and extrinsic neurons, including afferent terminals that enter the lobes.

Our data suggest that the number of extrinsic neurons is much smaller than the number of Kenyon cells. Enhancer-trap analysis suggests that the Kenyon cells are a biochemically heterogeneous population (Yang et al. 1995; Ito et al. 1997a). Although essentially all the Kenyon cell fibers extend through the lengths of each lobe, certain biochemically distinct subtypes occupy characteristic trajectories that can be resolved in transverse sections. Others appear to distribute isomorphically across the lobes. It is likely that extrinsic neurons that have processes extending among discrete subsets of Kenyon cells form synapses with a specific biochemical subtype of Kenyon cells. Extrinsic cells that have more diffuse processes across the lobes may interact with a relatively heterogeneous population of Kenyon cells. Such differences would be expected to contribute to the types of information each extrinsic neuron conveys.

PROJECTIONS OF MUSHROOM BODY EXTRINSIC NEURONS IN THE PROTOCEREBRUM

With the exception of a few connections to

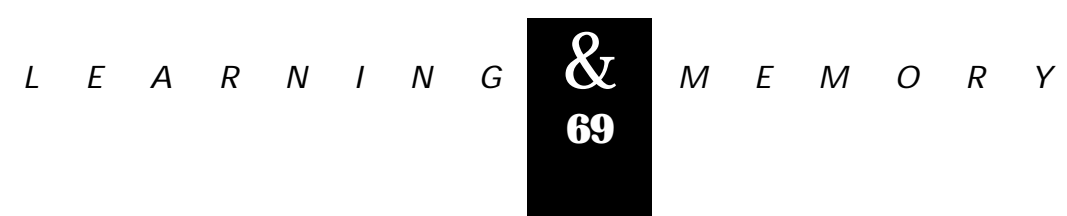


Ito et al.

the ventral body and the lateral horn, all extrinsic neurons so far identified have connections to the anterior part of the $\mathrm{s} \mathrm{m} \mathrm{pr}, \mathrm{s} I \mathrm{pr}$, and i $\mathrm{m} \mathrm{pr}$. This suggests that these areas (termed collectively here as "mushroom body-linked neuropils") are the primary neuropils to and from which the mushroom body lobes send and receive information (Fig. 8). This is consistent with the observations on the bee and cockroach brains (Rybak and Menzel 1993; Li and Strausfeld 1997a,b).

Reduced silver stains show that the mushroom body-linked neuropils have quite complex structures, each comprising a delineated neuropil, the characteristic cellular organization of which is as yet little understood. The s $\mathrm{m}$ pr is supplied richly by ascending terminals of median bundle neurons, many of which originate in the tritocerebrum and suboesophageal ganglia (Musca domestica; Strausfeld 1976). Others ascend via the median bundle from the digestive tract (Duve et al. 1983). Neurons that descend from the cortex of the pars intercerebralis and the sI pr into the median bundle, or into the corpora cardiaca nerves, also have extensive processes in the s m pr (Strausfeld 1976).

Discussions about the supposed function of the mushroom bodies in learning and memory have invoked the possible existence of reinforcing or reverberating circuits mediated by feedback neurons from the lobes to the calyces, such as those described from honeybees (Gronenberg 1987; Rybak and Menzel 1993). Whereas we found no evidence for recurrent neurons in Drosophila, we do not exclude that, as in the cockroach ( $\mathrm{Li}$ and Strausfeld 1997a,b), certain neurons originating in the mushroom body-linked neuropils might project to the calyces, thereby forming an indirect feedback loop.

The significance of the efferent neuron projections from the mushroom bodies must be considered with respect to previous theories about the relationship of this neuropil to premotor pathways. Studies on various dipteran species, including Drosophila, demonstrate that the dendrites of descending neurons are confined mostly to the posterior slope in the deutocerebrum. In the blow fly Calliphora erythrocephala, two neurons were reported to connect the mushroom bodies and the posterior slope (Strausfeld et al. 1984). Whereas we found various other types of connections from the mushroom bodies to nearby neuropils, none projected to the posterior slope. The paucity of direct projections to the posterior slope has been reported already in bees and cockroaches (Rybak and Menzel 1993; Li and Strausfeld 1997a,b). Thus, although we cannot exclude that a few neurons might have escaped our detection (and that further screening might reveal them), it is more likely that, should it exist, a direct connection to descending neurons would not represent the major efferent pathway from the mushroom bodies.

\section{IMPLICATION OF MUSHROOM BODY EXTRINSIC CELL RELATIONSHIPS IN THE CONTEXT OF ELEVATED LEVELS OF LEARNING-RELATED MOLECULES}

Our data suggest that the posterior slope is not the main target of the mushroom body efferents and that it is more plausible that mushroom bodies communicate with descending pathways indirectly, via intervening networks of local and relay interneurons in the protocerebrum. Thus, if the mushroom bodies serve as centers for learning and memory, as has often been proposed, they would have to gate descending pathways via several intermediate interneurons. On the other hand, because the mushroom bodies are closer to the sensory input than to the motor output (Fig. 8), it is reasonable to suggest an alternative role for them-namely, that the mushroom bodies serve as preprocessors that provide modified olfactory information to subsequent higher-order centers in the protocerebrum and that it is these centers that gate descending pathways conditionally.

In the following, we will discuss whether anatomical observations that best support this latter interpretation can be obviated by molecular and functional studies that have been used to support the notion that mushroom bodies are learning and memory centers.

Molecular studies report that mushroom bodies have elevated or exclusive concentrations of biochemicals associated with learning and memory (Nighorn et al. 1991; P.L. Han et al. 1992; Skoulakis et al. 1993; K.-A. Han et al. 1996; Skoulakis and Davis 1996; Muller 1997; Grotewiel et al. 1998). These studies seem to support the notion that mushroom bodies are the center for learning and memory, and from them it could be reasoned that if the mushroom bodies are merely preprocessors, then the hypothesized subsequent centers for learning and memory should at least be as prominently labeled as the mushroom bodies.

Specific labeling of mushroom bodies, however, raises an interesting problem of interpreta-

$$
\begin{array}{llllllllllllllll}
L & E & A & R & N & I & N & G & \underset{70}{\mathbf{X}} & M & E & M & O & R & Y
\end{array}
$$


tion. The Drosophila mushroom bodies appear to play a role only in olfactory learning, because mushroom body ablation does not affect visual learning (deBelle and Heisenberg 1994). But if specific labeling by learning-related molecules reveals a special structure that underlies one type of learning and memory (namely, olfactory memory), then why are other neuropil regions, elsewhere in the central brain, underlying nonolfactory learning and memory not similarly labeled? Thus, if it is proposed that learning-related molecules are concentrated in, or are exclusive to, the mushroom bodies, how do other learning-related neuropil regions perform their task without these substances? If these regions actually contain significant levels of such molecules, why are they not labeled as prominently as the mushroom bodies? If such nonolfactory learning centers have so far escaped detection, is it not also possible that higher-order olfactory learning neuropils subsequent to the mushroom bodies might have escaped detection?

One reason for this paradox can be ascribed to the structural peculiarity of the mushroom bodies. Nowhere else in the insect brain do such numerous fibers run in a parallel and coherent manner. The distinctive profile of the mushroom body is obvious even when as few as $5 \%$ of the 3000 Kenyon cells are stained. Because the diameters of the thinnest Kenyon cell axons are at the limit of optical resolution, if labeled and unlabeled fibers are mingled, they cannot be resolved separately even with the best light microscope. This can lead to the misinterpretation that all the mushroom body is labeled when the gene of interest is actually expressed in only a subset of Kenyon cells (e.g., Fig. 4E and F).

The coherent anatomy, and thus subjective prominence of the mushroom bodies, can lead observers to disregard the existence of labeled cells in other neuropils. For example, essentially all the mushroom body-specific GAL4 enhancer-trap strains described so far show expression in various other neurons of the central brain ( $Y$ ang et al. 1995; Connolly et al. 1996; Ito et al. 1997a). However, the scattered distribution of cell bodies and dispersed fiber structures make the labeling of these cells much less pronounced, even when the number of labeled cells outside the mushroom bodies might even exceed the number of cells labeled within them.

Significantly, staining for a relatively ubiquitous chemical can be artifactual in that the mushroom bodies appear to be revealed preferentially. When the nuclear-specific reporter NLS-lacZ gene is expressed in all the neurons by the GAL4 gene under the neuron-specific promoter elav (Luo et al.
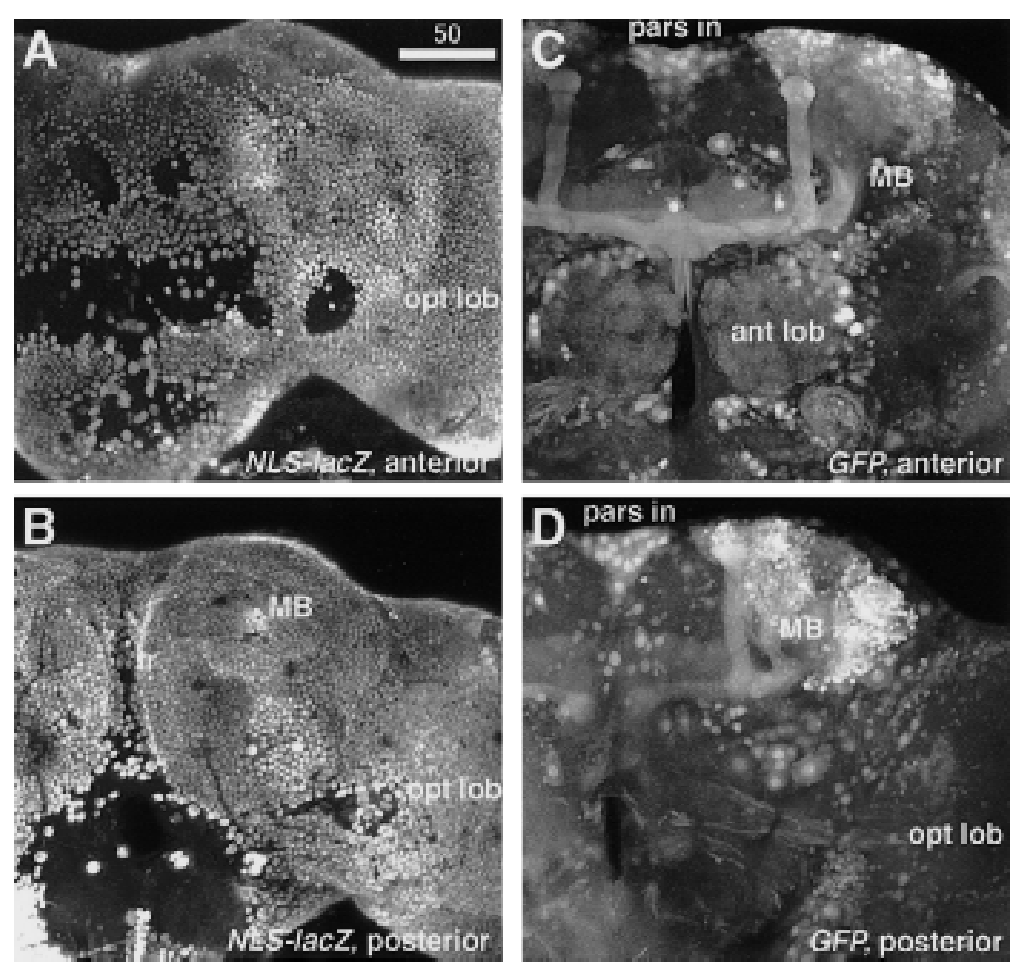

Figure 9: Artifactual mushroom bodypreferential labeling caused by ubiquitous cytoplasmic staining. Confocal reconstruction of 120-140 optical sections of the brain of the elav-GAL4 strain. The elav promoter drives GAL4 in all the neurons. With the nuclear-specific reporter UAS-NLS-lacZ, all the cell bodies are clearly labeled $[(A)$ anterior; $(B)$ posterior]. The empty holes are regions where the cortex is so thin that there are no neuronal cell bodies. With the cytoplasmic GFP reporter, the mushroom body stains intensely compared to other neuropil regions [ $(C)$ anterior; $(D)$ posterior]. The pars intercerebralis (pars in), antennal lobes (ant lob), and some tracts from the optic lobes (opt lob) are also labeled stronger. Amorphous and ubiquitous staining in other brain regions can easily be misinterpreted as background. 
Ito et al.

1994), ubiquitous staining is observed (Fig. 9A,B). When the cytoplasm of these cells is labeled by the cytoplasmic reporter GFP, staining in the mushroom bodies appears elevated greatly (Fig. 9C,D). Interestingly, the elevation occurs not only at the whole structural level but also at the single-cell level; each Kenyon cell body appears as if it were labeled more strongly than any other neuron in the brain. Such elevated staining in the mushroom bodies was also observed with other ubiquitous GAL4 drivers such as actin promoter and many enhancertrap strains with nonspecific GAL4 expression, and with other cytoplasmic reporter genes such as lacZ and tau.

There can be several reasons for such apparent elevation of the signal. First, the Kenyon cells may have higher levels of activity than other neurons. Second, the coherent anatomy of the pedunculus and lobes makes it easier to detect labeling in these structures. And third, Kenyon cells have much smaller cell volumes than other neurons. As show $n$ in Figure 9B, Kenyon cell bodies are among the smallest in the brain, and the volume of the mushroom body neuropil is also small. A preliminary analysis of serial paraffin sections revealed that the volume of the Drosophila mushroom body neuropil accounts for only $2.5 \%$ of the central brain's total neuropil volume (sub- and supraoesophageal ganglia without optic lobes). The number of cells in the Drosophila central brain is roughly estimated to be $\sim 15,000-20,000$ for each hemisphere (N. Strausfeld, unpubl.). Thus, each mushroom body's 3000 Kenyon cells comprise $15 \%-20 \%$ of the central brain's neurons packed into $<3 \%$ of the neuropil volume. Kenyon cells have been estimated to comprise the highest density of neurons of any known brain (see Strausfeld 1976). If gene activity is at the same level in a neuron regardless of its size, the same amount of mRNA and protein molecules would be more concentrated in a minute Kenyon cell than in a larger neuron.

In reality, gene activity may vary among neurons, since the production of many proteins is controlled by feed-back mechanisms. If the gene activity is controlled according to the intracellular protein concentration per cytoplasmic volume, Kenyon cells will not show elevated staining. If, how ever, the activity of certain genes is controlled according to the density of the gene product per membrane area, elevated staining should be seen in Kenyon cells. A high density of small neurons implies a correspondingly large amount of membrane within a small volume of neuropil. For ex- ample, membrane-bound cyclic AMP-dependent protein kinase type II (PKA) shows elevated staining in the mushroom bodies. Significantly, other neuropil regions are also stained with the antibody against PKA (Muller 1997), and the neuropils with higher levels of PKA staining generally consist of small neurons or fine arborizations (e.g. optic lobes and antennal lobe glomeruli) which, presumably, have higher membrane densities. In the mushroom bodies, the $\beta$ lobe, which has the thinnest axons and thus higher membrane density, is more strongly labeled than $\gamma$. The amount of membranebound molecules are not necessarily regulated according to area density, however. Antibodies against certain ubiquitous membrane antigens do not show elevated staining in the mushroom bodies (Nighorn et al. 1991).

Naturally, expression of many learning-related genes does show cell-type specific regulation. Even in these cases, how ever, smaller cell volume, large cell number, coherent organization, and higher membrane density of Kenyon cells might reveal the label in the mushroom bodies as a disproportionately stronger signal than in non-Kenyon cells with comparable levels of gene expression. It might therefore be expected that staining for learning-related substances may appear less prominent in structures other than the mushroom bodies, unless the labeled neurons are tightly packed or their gene expression is significantly upregulated. Most reports on learning-related genes indeed mention w eaker expression in brain areas outside the mushroom bodies. Detailed examination of these areas might reveal higher-order centers for olfactory memory, as well as memory centers for visual and mechanosensory association.

\section{IMPLICATION OF MUSHROOM BODY EXTRINSIC CELL RELATIONSHIPS IN LEARNING AND MEMORY}

The central role of the mushroom bodies in learning and memory has also been suggested by various functional studies. Can these results be explained by regarding the mushroom body as an intermediate preprocessor rather than the center?

One of the reasons why associative learning and memory is ascribed specially to the mushroom bodies is that the disruption or ablation of this structure affects selectively these functions yet maintains the fly's ability to discriminate different odors and to perform nonlearning associations (Heisenberg 1980; Heisenberg et al. 1985; de Belle

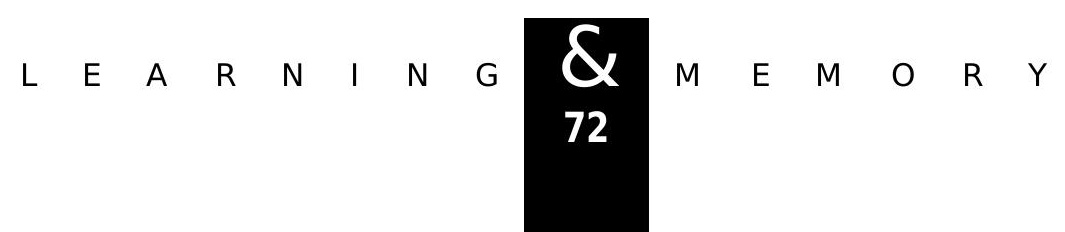




\section{MUSHROOM BODY EXTRINSIC NEURONS}

and Heisenberg 1994). Why the discriminating function is not disturbed may be explained by the fact that chemosensory information is transferred not only to the calyces but also to the lateral horns, which are not affected by mutants and ablation experiments. The learning and memory phenotype can be explained as well without regarding the mushroom bodies as the center for these functions. If an intermediate preprocessor that relays information to subsequent learning and memory centers is disrupted or ablated, higher-order centers to which it relays cannot receive signals necessary for their function.

By the same logic, a necessity for a larger preprocessor for handling more complicated signals would explain why changes in mushroom body volume are associated with specific behavioral tasks (Withers et al. 1993, 1995; Heisenberg et al. 1995; Gronenberg et al. 1996) or why, among a species of social Hymenoptera, castes that have complex multitask behaviors appear to have the largest mushroom bodies (Dujardin 1850; von Alten 1910).

Selective lesions and focal cooling of the mushroom body lobes cause perturbation and abolition of memories in ants, bees, and cockroaches (Vowles 1964; Erber et al. 1980; Mizunami et al. 1993). The lesions would perturb not only the internal information processing of the mushroom bodies but also information transfer from the mushroom body lobes to the next higher-order protocerebral associative centers. Focal cooling would affect the activity of not only the lobes but also the surrounding neuropils which, by analogy with Drosophila anatomy, might receive output from the mushroom bodies. Thus, these results can be understood without ascribing a memory function to the mushroom bodies themselves.

The possible role of other protocerebral neuropils in learning and memory functions is suggested by recent experiments in which ectopic expression of a mutant form of the catalytic $\alpha$-subunit of a G-protein, which interferes with normal CAMP signaling and hence perturbs associative memory, causes defective olfactory conditioning (Connolly et al. 1996). The defects were observed when the expression of the mutant subunit is driven in the mushroom bodies. Although this appears to confirm their central role in olfactory conditioning, the enhancer-trap strains used for this experiment drive expression not only in Kenyon cells but also in neurons that project to the anterior s $\mathrm{m} \mathrm{pr}$ and i $\mathrm{m} \mathrm{pr}$; that is, those areas receiving mushroom body efferents.

\section{POSSIBLE ROLES OF MUSHROOM BODIES IN OLFACTORY PROCESSING}

Thus, our present anatomical data, and much of the published biochemical and functional data, can be interpreted by regarding the mushroom bodies as a preprocessor that provides entry to higher learning and memory centers. In Drosophila, the sensory input to the calyces is limited primarily to olfactory and gustatory signals, preprocessed in the antennal lobe and sent to each calyx via the iACT. Our results show that the medial ( $\beta$ and $\gamma$ ) and vertical ( $\alpha$ and $\alpha^{\prime}$ ) lobes, but not pedunculus, receive additional afferents from surrounding neuropils. Thus, we predict that olfactory information carried by the Kenyon cells from the calyces is integrated with multimodal inputs at the lobes.

Convergence of multimodal information in the mushroom bodies has been suggested by functional studies in other species, although this has yet to be show $n$ in Drosophila. For example, multimodal response of efferent neurons have been show $n$ by intracellular recordings from the cockroach Periplaneta americana ( $\mathrm{Li}$ and Strausfeld 1997a,b). Like Drosophila, cockroach mushroom bodies have a massive olfactory supply to the calyces. How ever, efferents from the mushroom bodies respond to olfactory as well as visual, acoustic, and mechanosensory olfactory stimuli. The reason for this is because there are numerous afferents from sensory neuropils to the lobes, some of which carry multimodal information ( $\mathrm{Li}$ and Strausfeld 1997b). Structurally, these afferents are similar in appearance to those shown here for Drosophila. And, because inputs to the lobes do not form tightly bound tracts, they are not anatomically obvious unless each is selectively filled with dye. To reveal information pathways from visual and mechanosensory neuropils to the mushroom body lobes of Drosophila brains, further screening of Golgi preparations and GAL4 strains would be important.

Thus, with consideration to the present findings in Drosophila and their similarity with studies on other insect species, a plausible alternative role for the mushroom bodies might be as follows. Because visual, acoustic, and tactile information is not relayed to the antennal lobes, it is likely that che-

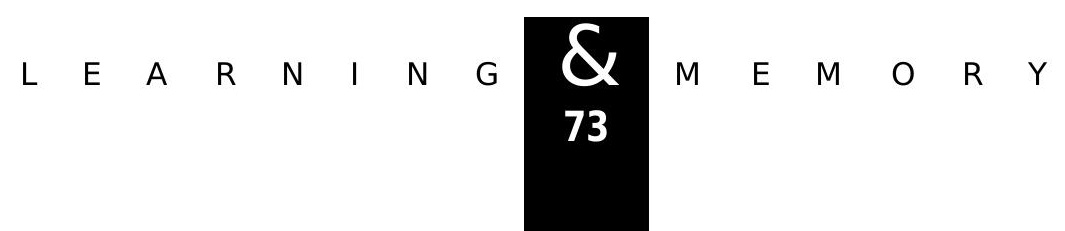


Ito et al.

mosensory signals relayed from the antennal lobes to the calyces are independent of other ambient sensory signals. Afferents supplying the mushroom body lobes add multimodal information to olfactory signals, thus providing a multimodal context to olfactory information that is then relayed by efferent neurons to higher protocerebral regions for further processing. If the combination of olfactory signals with other modalities involves modification of synaptic efficacy, as is suggested by recordings of efferent neurons of bees during olfactory conditioning (Mauelshagen 1993), then it is reasonable to suppose that molecules relating to synaptic plasticity would be found abundantly in the mushroom bodies as elsewhere in the brain.

In conclusion, previous biochemical and functional studies can be interpreted equally well by assuming or by rejecting a central role of the mushroom bodies in learning and memory. Whereas current knowledge is not sufficient to support either possibility, our present anatomical data suggest that the mushroom bodies function in close collaboration with the neighboring mushroom bodylinked neuropils. It is therefore crucial that the search for neuropils underlying learning and memory is expanded to include those regions.

\section{Acknowledgments}

We thank Joachim U rban and Gerhard Technau for $M z$ series enhancer-trap strains, Barry Dickson for the UAS-GFP S65T strain, and Yasushi Hiromi for UAS-NLS lacZ strain. We also thank Maiko $U$ e and Tsuyoshi $O$ tsuka for screening GAL4 enhancer-trap strains, and Wakae Awano for maintenance of the stocks. We are grateful to Reinhard Stocker, Martin Heisenberg, and U we Homberg for helpful discussion. Robert Gomez provided invaluable technical assistance. Ilya Vilinsky generated some of the Golgi preparations and made drawings of Kenyon cells used in this study. Support for this work came from operational funding to the ERATO Yamamoto Behavior Genes Project (D.Y.), from a National Science Foundation grant IBN -931629 (N.J.S.), from a Human Frontier Science Program grant (M.R., with two others), and from the Sloan and McKnight Foundations (M.R.).

The publication costs of this article were defrayed in part by payment of page charges. This article must therefore be hereby marked "advertisement" in accordance with 18 USC section 1734 solely to indicate this fact.

\section{References}

Balling, A., G.M. Technau, and M. Heisenberg. 1987. Are the structural changes in adult Drosophila mushroom bodies memory traces? Studies on biochemical learning mutants. J. Neurogenet. 4: 65-73.

Barth, M. and M. Heisenberg. 1997. Vision affects mushroom bodies and central complex in Drosophila melanogaster. Learn. \& Mem. 4: 219-229.

Bicker, G. and I. Hähnlein. 1995. NADPH-diaphorase expression in neurones and glial cells of the locust brain. NeuroReport 6: 325-328.

Bodian, D. 1936. A new method for staining nerve fibers and nerve endings in mounted paraffin sections. Anat. Rec. 69: 153-162.

Brand, A.H. and N. Perrimon. 1993. Targeted gene expression as a means of altering cell fates and generating dominant phenotypes. Development 118: 401-415.

Connolly, J.B., I.J.H. Roberts, J.D. Armstrong, K. Kaiser, M. Forte, T. Tully, and C.J. O'Kane. 1996. Associative learning disrupted by impaired Gs signaling in Drosophila mushroom bodies. Science 274: 2104-2107.

Davis, R.L. 1993. Mushroom bodies and Drosophila learning. Neuron 11: 1-14.

deBelle, J.S. and M. Heisenberg. 1994. Associative odor learning in Drosophila abolished by chemical ablation of mushroom bodies. Science 263: 692-695.

DiAntonio, A., R.W. Burgess, A.C. Chin, D.L. Deitcher, R.H. Scheller, and T.L. Schwarz. 1993. Identification and characterization of Drosophila genes for synaptic vesicle proteins. J. Neurosci. 13: 4924-4935.

Dujardin, F. 1850. Memoire sur le systeme nerveux des insectes. Ann. Sci. Natl. Zool. 14: 195-206.

Durst, C., S. Eichmuller, and R. Menzel. 1994. Development and experience lead to increased volume of subcompartments of the honeybee mushroom body. Behav. Neural. Biol. 62: 259-263.

Duve, H., A. Thorpe, and N.J. Strausfeld. 1983. Cobalt-immunocytochemical identification of peptidergic neurons in Calliphora innervating central and peripheral targets. J. Neurocytol. 12: 847-861.

Elphick, M.A., R.C. Rayne, V. Riveros-M oreno, A. Moncada, and M. O'Shea. 1995. Nitric oxide synthase in locust olfactory interneurons. J. Exp. Biol. 198: 821-829.

Erber, J., T. Masuhr, and R. Menzel. 1980. Localization of short-term memory in the brain of the bee, Apis mellifera. Physiol. Entomol. 5: 343-358.

Estes, P., J. Roos, A. van der Bliek, R.B. Kelly, K.S. Krishnan, and M. Ramaswami. 1996. Traffic of dynamin within single Drosophila synaptic boutons relative to compartment-specific presynaptic markers. J. Neurosci. 16: 5443-5456.

Ferveur, J.F., K.F. Stortkuhl, R.F. Stocker, and R.J. Greenspan. 1995. Genetic feminization of brain structures and changed sexual orientation in male Drosophila. Science 267: 902-905. 


\section{MUSHROOM BODY EXTRINSIC NEURONS}

Golic, K.G. and S. Lindquist. 1989. The FLP recombinase of yeast catalyzes site-specific recombination in the Drosophila genome. Cell 59: 499-509.

Gronenberg, W. 1987. Anatomical and physiological properties of feedback neurons of the mushroom bodies in the bee brain. Exp. Biol. 46: 115-125.

Gronenberg, W., J.J. Milde, and N.J. Strausfeld. 1995. O culomotor control in calliphorid flies: Multimodal descending neurons converging at frontal neck motor neuropil. J. Comp. Neurol. 361: 267-284.

Gronenberg, W., S. Heeren, and B. Hölldobler. 1996. Age-dependent and task-related morphological changes in the brain and the mushroom bodies of the ant Camponotus floridanus. J. Exp. Biol. 199: 2011-2019.

Grotewiel, M.S., C.D.O. Beck, K.H. Wu, X.-R. Zhu, and R.L. Davis. 1998. Integrin-mediated short-term memory in Drosophila. Nature 391: 455-460.

Hall, J.C. 1979. Control of male reproductive behavior by the central nervous system of D rosophila: Dissection of a courtship pathway by genetic mosaics. Genetics 92: 437-457.

Han, K.-A., N.S. Millar, M.S. Grotewiel, and R.L. Davis. 1996. DAMB, a novel dopamine receptor expressed specifically in Drosophila mushroom bodies. Neuron 16: 1127-1135.

Han, P.L., L.R. Levin, R.R. Reed, and R.L. Davis. 1992. Preferential expression of the Drosophila rutabaga gene in mushroom bodies, neural centers for learning in insects. Neuron 9: 619-627.

Heisenberg, M. 1980. Mutants of brain structure and function: What is the significance of the mushroom bodies for behavior? In Development and neurobiology of Drosophila (ed. Q. Siddiqi), pp. 373-390. Plenum, N ew York, NY.

Heisenberg, M., A. Borst, S. Wagner, and D. Byers. 1985. Drosophila mushroom body mutants are deficient in olfactory learning. J. Neurogenet. 2: 1-30.

Heisenberg, M., M. Heusipp, and C. Wanke. 1995. Structural plasticity in the Drosophila brain. J. Neurosci.

15: 1951-1960.

Hinke, W. 1961. Das relative postembryonale Wachstum der Hirnteile von Culex pipiens, Drosophila melanogaster und Drosophila mutanten. Z. Morph. Ö kol. Tiere 50: 81-118.

Homberg, U., R.A. Montague, and J.G. Hildebrand. 1989. Anatomy of antenno-cerebral pathways in the brain of the sphinx moth Manduca sexta. Cell Tissue Res. 254: 255-281.

Hurd, D.D. and W.M. Saxton. 1996. Kinesin mutations cause motor neuron disease phenotypes by disrupting fast axonal transport in Drosophila. Genetics 144: 1075-1085.
Ito, K. and Y. Hotta. 1992. Proliferation pattern of postembryonic neuroblasts in the brain of Drosophila melanogaster. Dev. Biol. 149: 134-148.

Ito, K., J. U rban, and G.M. Technau. 1995. Distribution, classification, and development of Drosophila glial cells in the late embryonic and early larval ventral nerve cord. Wilhelm Roux's Arch. Dev. Biol. 204: 284-307.

Ito, K., W. Awano, K. Suzuki, Y. Hiromi, and D. Yamamoto. 1997a. The Drosophila mushroom body is a quadruple structure of clonal units each of which contains a virtually identical set of neurones and glial cells. Development 124: 761-771.

Ito, K., H. Sass, J. U rban, A. H ofbauer, and S. Schneuwly. 1997b. GAL4-responsive UAS-tau as a tool for studying the anatomy and development of the Drosophila central nervous system. Cell Tissue Res. 290: 1-10.

Jäger, R.J. and K.-F. Fischbach. 1987. Some improvements of the Heisenberg-Böhl method for mass histology of Drosophila heads. Dros. Inf. Serv. 66: 162-165.

Kenyon, F.C. 1896. The meaning and structure of the so-called "mushroom bodies" of the hexapod brain. Amer. Nat. 30: 643-650.

King, D.G. and K.L. Valentino. 1983. On neuronal homology: A comparison of similar axons in Musca, Sarcophaga and Drosophila (Diptera: Schizophora). J. Comp. Neurol. 219: 1-9.

Li, Y.-S. and N.J. Strausfeld. 1997a. Morphology and sensory modality of mushroom body efferent neurons in the brain of the cockroach, Periplaneta americana. J. Comp. Neurol. 387: 631-650.

- - - 1997b. Afferents supplying insect mushroom bodies carry multimodal sensory information. Soc. Neurosci. Abstr. 23: 1571.

Luo, L., Y.J. Liao, L.Y. Jan, and Y.N. Jan. 1994. Distinct morphogenetic functions of similar small GTPases: Drosophila Dracl is involved in axonal outgrowth and myoblast fusion. Genes \& Dev. 8: 1787-1802.

Mauelshagen, J. 1993. Neural correlates of olfactory learning paradigms in an identified neuron in the honey bee brain. $J$. Neurophys. 69: 609-625.

Meinertzhagen, I.A. and S.D. O'N eil. 1991. Synaptic organization of columnar elements in the lamina of the wild type in Drosophila melanogaster. J. Comp. Neurol. 305: 232-263.

Milde, J.J. and N.J. Strausfeld. 1990. Cluster organization and response characteristics of the giant fibre pathway of the blow fly Calliphora erythrocephala. J. Comp. Neurol. 294: 59-75.

Mizunami, M., J.M. Weibrecht, and N.J. Strausfeld. 1993. A new role for the insect mushroom bodies: Place memory and 
Ito et al.

motor control. In Biological neural networks in invertebrate neuroethology and robotics (ed. R.D. Beer, R. Ritzmann, and T. McKenna), pp. 199-225. Academic Press, Cambridge, MA.

Mobbs, P.G. 1982. The brain of the honeybee Apis mellifera. 1. The connections and spatial organizations of the mushroom bodies. Phil. Trans. R. Soc. Lond. B

298: 309-354.

Muller, U. 1997. Neuronal CAM P-dependent protein kinase type II is concentrated in mushroom bodies of Drosophila melanogaster and the honeybee Apis mellifera. J. Neurobiol. 33: 33-44.

Nässel, D.R. and K. Elekes. 1992. Aminergic neurons in the brain of blow flies and Drosophila: Dopamine- and tyrosine hydroxylase-immunoreactive neurons and their relationship with putative histaminergic neurons. Cell Tissue Res. 267: 147-167.

Nayak, S.V. and R.N. Singh. 1983. Sensilla on the tarsal segments and mouthparts of Drosophila melanogaster M eigen (Diptera: Drosophilidae). Int. J. Insect Morphol. Embryol. 12: 273-292.

Nighorn, A., M.J. Healy, and R.L. Davis. 1991. The cyclic AMP phosphodiesterase encoded by the Drosophila dunce gene is concentrated in the mushroom body neuropil. Neuron 6: 455-467.

O'Dell, K.M., J.D. Armstrong, M.Y. Yang, and K. Kaiser. 1995. Functional dissection of the Drosophila mushroom bodies by selective feminization of genetically defined subcompartments. Neuron 15: 55-61.

Pearson, L. 1971. The corpora pedunculata of Sphinx ligustri L. and other Lepidoptera: An anatomical study. Phil. Trans. $R$. Soc. Lond B. 259: 477-516.

Phelan, P. and J.P. Bacon. 1997. The giant fiber pathway. Flybrain on-line. http://www.flybrain.org. Accession number AC00134.

Power, M.E. 1946. The antennal centers and their connections within the brain of Drosophila melanogaster. J. Comp. Neurol. 85: 485-518.

Prokop, A. and G.M. Technau. 1993. Cell transplantation. In Cellular interactions in development: A practical approach (ed. D.A. Hartley), pp. 33-57. Oxford U niversity Press, Oxford, UK.

Robertson, H.M., C.R. Preston, R.W. Phillis, D. Johnson-Schlitz, W.K. Benz, and W.R. Engels. 1988. A stable genomic source of $\mathrm{P}$ element transposase in Drosophila melanogaster. Genetics 118: 461-470.

Rybak, J. and R. Menzel. 1993. Anatomy of the mushroom bodies in the honey bee brain: The neuronal connections of the alpha-lobe. J. Comp. Neurol. 334: 444-465.

Singh, R.N. and S.V. Nayak. 1985. Fine structure and primary sensory projections of sensilla on the maxillary palp of
Drosophila melanogaster Meigen (Diptera: Drosophilidae). Int. J. Insect Morphol. Embryol. 14: 291-306.

Skoulakis, E.M.C. and R.L. Davis. 1996. Olfactory learning deficits in mutants for Leonardo, a Drosophila gene encoding a 14-3-3 protein. Neuron 17: 931-944.

Skoulakis, E.M.C., D. Kalderon, and R.L. Davis. 1993. Preferential expression in mushroom bodies of the catalytic subunit of protein kinase $A$ and its role in learning and memory. Neuron 11: 197-208.

Stocker, R.F. 1994. The organization of the chemosensory system in Drosophila melanogaster: A review. Cell Tissue Res. 275: 3-26.

Stocker, R.F., M.C. Lienhard, A. Borst, and K.-F. Fischbach. 1990. Neuronal architecture of the antennal lobe in Drosophila melanogaster. Cell Tissue Res. 262: 9-34.

Stopfer, M., S. Bhagavan, B.H. Smith, and G. Laurent. 1997. Impaired odour discrimination on desynchronization of odour encoding neural assemblies. Nature 390: 70-74.

Strausfeld, N.J. 1976. Atlas of an insect brain. Springer-Verlag, Berlin, Germany.

- - - 1998. The insect mushroom bodies: A uniquely identifiable neuropil. In Identified neurons in model systems (ed. J.L. Leonard). Harvard University Press, Cambridge, MA (In Press).

Strausfeld, N.J. and U.K. Bassemir. 1983. Cobalt-coupled neurons of a giant fibre system in Diptera. J. Neurocytol. 12: $971-991$.

Strausfeld, N.J. and W. Gronenberg. 1990. Descending neurons supplying the neck and flight motor of Diptera: O rganization and neuroanatomical relationships with visual pathways. J. Comp. Neurol. 302: 954-972.

Strausfeld, N.J. and I.A. Meinertzhagen. 1998. The insect neuron: Morphologies, structures and relationships to neuroarchitecture. In Microscopic anatomy of invertebrates (ed. F.W. Harrison and M. Locke), Vol. 11b, pp. 487-538. Wiley-Liss. N ew York, NY.

Strausfeld, N.J., U. Bassemir, R.N. Singh, and J.P. Bacon. 1984. O rganizational principles of outputs from dipteran brains. J. Insect Physiol. 30: 73-93.

Strausfeld, N.J., L. Hansen, Y.-S. Li, R.S. Gomez, and K. Ito. 1998. Evolution, discovery, and interpretations of arthropod mushroom bodies. Learn. \& Mem., (this issue).

Strauss, R. and M. Heisenberg. 1993. A higher control center of locomotor behavior in the Drosophila brain. J. Neurosci. 13: 1852-1861.

Tettamanchi, M., J.D. Armstrong, K. Endo, M.Y. Tang, K. Furukubo-Tokunaga, K. Kaiser, and H. Reichert. 1997. Early development of the Drosophila mushroom bodies, brain 
centers for associative learning and memory. Dev. Genes Evol. 207: 242-252.

Tolbert, L.P. and J.G. Hildebrand. 1981. O rganization and synaptic ultrastructure of glomeruli in the antennal lobes of the moth Manduca sexta: A study using thin sections and freeze-fracture. Proc. R. Soc. London Ser. B 213: 279-301.

Trimble, W.S., D.M. Cowan, and R.H. Scheller. 1988. vamp-1: A synaptic vesicle-associated integral membrane protein. Proc. Natl. Acad. Sci. 85: 4538-4542.

von Alten, H. 1910. Zur Phylogenie des

Hymenopterengehirns. Jena Z. Naturwiss. 46: 511-590.

Vowles, D.M. 1955. The structure and connections of the corpora pedunculata in bees and ants. Q. J. Microsc. Sci. 96: 239-255.

1964. Olfactory learning and brain lesion in the wood ant (Formica rufa). J. Comp. and Physiol. Psychol. 58: 105-111.

Withers, G.S., S.E. Fahrbach, and G.E. Robinson. 1993. Selective neuroanatomical plasticity and division of labour in the honeybee. Nature 364: 238-240.

- - - 1995. Effects of experience and juvenile hormone on the organization of the mushroom bodies of honey bees. $J$. Neurobiol. 26: 130-144.

Yang, M.Y., J.D. Armstrong, I. Vilinsky, N.J. Strausfeld, and K. Kaiser. 1995. Subdivision of the Drosophila mushroom bodies by enhancer-trap expression patterns. Neuron 15: 45-54.

Received January 27, 1998; accepted in revised form June 10, 1998. 


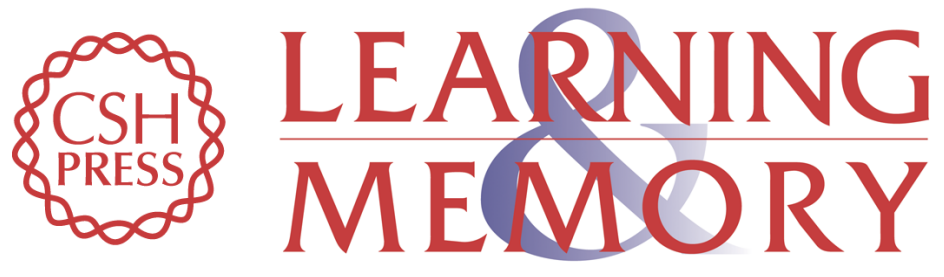

\section{The Organization of Extrinsic Neurons and Their Implications in the Functional Roles of the Mushroom Bodies in Drosophila melanogaster Meigen}

Kei Ito, Kazumi Suzuki, Patricia Estes, et al.

Learn. Mem. 1998, 5:

Access the most recent version at doi:10.1101//m.5.1.52

References This article cites 65 articles, 17 of which can be accessed free at: http://learnmem.cshlp.org/content/5/1/52.full.html\#ref-list-1

License

Email Alerting

Receive free email alerts when new articles cite this article - sign up in the box at the Service top right corner of the article or click here. 